MATHEMATICS OF COMPUTATION

Volume 70, Number 233 , Pages 395-417

S 0025-5718(00)01295-3

Article electronically published on July 21, 2000

\title{
CLASSIFICATION OF QUINTIC EUTACTIC FORMS
}

\author{
CHRISTIAN BATUT
}

\begin{abstract}
From the classical Voronoi algorithm, we derive an algorithm to classify quadratic positive definite forms by their minimal vectors; we define some new invariants for a class, for which several conjectures are proposed. Applying the algorithm to dimension 5 we obtain the table of the 136 classes in this dimension, we enumerate the 118 eutactic quintic forms, and we verify the Ash formula.
\end{abstract}

\section{INTRODUCTION}

In a given dimension $n$, there are only finitely many eutactic quadratic forms up to equivalence. This was first shown by Avner Ash (Ash1) by means of Morse theory: he gave a new interpretation of eutactic forms as the nondegenerate critical points of the function $\operatorname{det}(Q) / m(Q)^{n}$ on the symmetric positive definite forms, where $m(Q)$ is the minimum of $Q$ on integral nonzero vectors and $\operatorname{det}(Q)$ is its determinant. He also proved in Ash2] a "mass formula with signs" relating the orders of the automorphism groups of all integrally unequivalent eutactic forms. In a recent paper $(\overline{\mathrm{BM}}])$, A.-M. Bergé and J. Martinet gave another proof of Ash's theorem together with a method for the enumeration of eutactic forms: they classify the positive definite forms by the sets of their minimal vectors into a finite set of so called minimal classes or cells ordered via inclusion; they enlarge the notion of eutaxy by taking off the convexity it involves; and they eventually characterize in their classes the weakly eutactic forms as the ones (if any) at which the Hermite function $\gamma(Q)=m(Q) / \operatorname{det}(Q)^{1 / n}$ assumes its minimum (ensuring, by the convexity of this function, their uniqueness up to equivalence).

So the complete enumeration of eutactic forms can be based on the classification of forms, then on the study of the Hermite constant in each class. It was done up to dimension 4 by Bergé and Martinet using combinatorial methods and starting from the bottom, i.e., from classes of forms having exactly $n$ independent minimal vectors (looking for eutaxy, one need only consider forms whose minimal vectors span the space $\mathbb{R}^{n}$, see $\S 3$ ).

Actually, the cell classification as well as the determination of the minimum of $\gamma$ had been done previously for $n \leq 4$ by W. I. Štogrin in [Sto], a paper of which Bergé and Martinet were not aware. However, Sto] does not contain any interpretation in terms of eutaxy.

The combinatorial going-up method theorically leads to the classification of $n$ dimensional perfect forms (forms entirely determined by their minimum and their

Received by the editor February 13, 1997 and, in revised form, June 19, 1997.

2000 Mathematics Subject Classification. Primary 11H55, 11H56; Secondary 11E10.

Key words and phrases. Quadratic form, minimal vectors, eutactic form.

(C)2000 American Mathematical Society 
minimal vectors) whose classes are the top vertices of the graph of minimal classes. So it is no wonder that the combinatorial way becomes prohibitively laborious from dimension 5 on.

The main aim of this paper is to describe an algorithm to obtain the graph of all minimal classes in a given dimension.

This algorithm is derived from the well-known Voronoi algorithm for perfect forms, and starts from the perfect forms and the configuration of their minimal vectors. A refinement of the Voronoi theory (Theorem 2.4) asserts that by removing in a convenient manner some vectors from these configurations we obtain all classes of minimal vectors, going down step by step from perfect classes. Actually, such a method could produce thousands of subsets $S$ of $\mathbb{Z}^{n}$ (430 at the first step in the five-dimensional case), and it is important to be able to decide just which pairs of subsets are in the same orbits under $\mathrm{GL}_{\mathrm{n}}(\mathbb{Z}$ ) (and so correspond to equivalent forms). This question is discussed in the algorithmic Section 4.

Note that in Section 2 we introduce an efficient (and easy to check) invariant for a class, derived from the notion of characteristic determinants introduced by Korkine and Zolotareff: in the column "Spect" of Table 3 below for the five-dimensional case, we can point out very few coincidences.

Actually, the best invariant to identify a class is the unique (up to equivalence) weakly eutactic forms it usually contains. As it realizes the minimum of the Hermite function in the class (and therefore is algebraic), we can use a gradient method to get a good approximate of its matrix, and finish its determination by some LLLalgorithm. We ascertain the results, and decide whether the form is indeed eutactic, by a formal calculation of its eutactic coefficients. In order to compare eutactic forms having the same field of definition (and identify their classes), we had to adapt the usual algorithm to formal calculations over number fields.

Section 3 is devoted to weakly eutactic forms, and in particular gives partial answers to questions by A. Ash (in private discussions): the conjugates of such a form (when it is not rational) are studied in Proposition 3.4, and a connection between classes without weakly eutactic forms and classes with weakly eutactic noneutactic forms arises in Theorem 3.2. This correspondence, together with results for dimension five, suggest another Euler relation, involving all classes instead of that of eutactic forms. Such a relation has just been proved by Christophe Bavard.

We also prove in Section 3 that each weakly eutactic form achieves the maximal automorphism group in its class, i.e., the stabilizer in $\mathrm{GL}_{\mathrm{n}}(\mathbb{Z})$ of the set $S$ of minimal vectors ("group of $S$ "). This group is a good invariant of the class (its order, together with the "spectrum" alluded to above, allows us to identify the 5-dimensional classes). Anyhow, as the motivation for this work was to check Ash's mass formula, we had to compute it even in the algebraic case by using an algorithm adapted to formal calculations.

The last section presents the complete results for quintic forms. Starting from the three known perfect forms and their Voronoi domains, this algorithm produces 136 minimal classes listed with their invariants in Table 3, and 118 eutactic forms (for the rational ones, see Table 4). This enumeration cannot contain a unique error as it confirms Ash's formula!

The amount of calculation increases rapidly with the dimension and, moreover, the perfect forms are known only up to dimension 7 (and out of reach beyond, as we know more than ten thousand 8-dimensional perfect forms). Moreover, the ratio of 
eutactic forms to perfect forms for dimension 5 makes us believe that there should exist many eutactic forms in dimensions greater than 5 .

\section{Minimal Classes}

Let us recall some definitions and notation. A positive definite quadratic form in $n$ variables

$$
Q(x)=\sum_{1 \leq i, j \leq n} a_{i j} x_{i} x_{j}, \quad x={ }^{t}\left(x_{1}, x_{2}, \cdots, x_{n}\right) \in \mathbb{Z}^{n},
$$

can be identified with its $n \times n$ symmetric positive definite matrix $A=\left(a_{i j}\right)$ with real entries, and we have $Q(x)={ }^{t} x A x$. Note that $Q(x)$ can be interpreted as the trace of the matrix $A x^{t} x$. We introduce on the $N$-dimensional vector space $V$ of $n \times n$ symmetric matrices with real entries, where

$$
N=n(n+1) / 2,
$$

the inner product

$$
\langle A, B\rangle=\operatorname{Tr}(A B),
$$

so that we have

$$
Q(x)=\left\langle A, x^{t} x\right\rangle .
$$

Two forms $Q$ and $Q^{\prime}$ will be said to be integrally equivalent if there is a matrix $U$ in $G L(n, \mathbb{Z})$ and a positive scalar $\lambda$ such that $Q^{\prime}(x)=\lambda Q(U x)$, or matricially $A^{\prime}=\lambda^{t} U A U$. We denote by $\operatorname{Aut}(Q)=\operatorname{Aut}(A)$ the stabilizer of $A$ in $\mathrm{GL}_{\mathrm{n}}(\mathbb{Z})$.

The minimum $m(Q)=m(A)$ on integral vectors is attained on a finite subset $S(Q)=S(A)$ of $\mathbb{Z}^{n}$. We denote by $s=|S(Q)| / 2$ the number of pairs $\pm x$ of minimal vectors.

The form $Q$ is said to be perfect if the matrices $x^{t} x, x \in S(Q)$, span the whole space $V$.

2.1 Definition. We call two forms $Q$ and $Q^{\prime}$ minimally equivalent if there is a form integrally equivalent to $Q^{\prime}$ with same set of minimal vectors as $Q$.

Two integrally equivalent forms are therefore minimally equivalent, and the notions of minimal and integral equivalences coincide if and only if the forms are perfect.

Since $S\left({ }^{t} U A U\right)=U^{-1} S(A)$, we can attach to each minimal class an orbit of a finite subset $S$ of $\mathbb{Z}^{n}$ under $\mathrm{GL}_{\mathrm{n}}(\mathbb{Z})$. Conversely, such an orbit corresponds to one class at most.

By using reduction arguments, one can prove that there are only finitely many classes (cf $[\mathrm{BM}]$ ).

Inclusion induces a partial ordering on the set of classes:

2.2 Definition. Let $\mathcal{C}_{1}$ and $\mathcal{C}_{2}$ be two minimal classes. We note $\mathcal{C}_{1} \prec \mathcal{C}_{2}$ if there exists $A_{1} \in \mathcal{C}_{1}$ and $A_{2} \in \mathcal{C}_{2}$ with $S\left(A_{1}\right) \subset S\left(A_{2}\right)$.

Let $A$ be a symmetric positive definite matrix with set of minimal vectors $S$ and minimum $m=1$. Its minimal class $\mathcal{C}$ can be represented up to integral equivalence by the set of symmetric positive definite matrices

$$
\mathcal{C}_{S}=\{M \mid S(M)=S \text { and } m(M)=1\} .
$$

By abuse of language we shall often refer to this set as the class of $A$. 
It is an open convex polyhedron in the linear variety

$$
\left\{M \mid \forall x \in S\left\langle M, x^{t} x\right\rangle=1\right\}=A+Y,
$$

where $Y$ is the orthogonal complement to the vector subspace $X$ spanned by the $x^{t} x, x \in S$.

The dual polyhedron of the class $\mathcal{C}_{S}$ is the convex hull $\mathcal{D}_{S}$ of the $x^{t} x, x \in S$, which is the classical Voronoi domain common to all matrices $A \in \mathcal{C}_{S}$. Its dimension in the $N$-dimensional space $V$ of symmetric $n \times n$ real matrices

$$
r=\operatorname{dim}\left(\mathcal{D}_{S}\right)=\operatorname{codim}\left(\mathcal{C}_{S}\right)
$$

is called the rank of perfection of the class $\mathcal{C}$ of $A$ (it is indeed an invariant for $\mathcal{C}$ ). From the description of $\mathcal{C}_{S}$ we see that the rank of perfection is a strictly increasing function on the set of minimal classes. We have $r \leq N$, and equality holds if and only if the class is perfect. Thus the dimension $N-r$ of the class $\mathcal{C}_{S}$ will be referred to as the defect of perfection.

In what follows, we will focus on the classes for which there are positive definite matrices in the corresponding Voronoi domain:

2.3 Definition. We call the classes associated to sets $S$ spanning $\mathbb{R}^{n}$ well rounded.

Voronoi proved $([\overline{\mathrm{Vor}}])$ that the Voronoi domain of a given symmetric positive definite matrix $A$ is an $r$-dimensional face of the Voronoi domain of some perfect form $P$. It follows that it is the intersection of $N-r$ hyperplane faces of the domain of $P$ (an algorithm for these faces is given in subsection 4.1). So, the minimal class $\mathcal{C}_{S}$ can be parametrized as follows:

$$
\mathcal{C}_{S}=\left\{P+t_{1} F_{1}+t_{2} F_{2}+\cdots+t_{N-r} F_{N-r}\right\},
$$

where $F_{1}, F_{2} \cdots F_{N-r} \in V$ are orthogonal to hyperplane faces of the Voronoi domain of $P$, and the $t_{i} \in \mathbb{R}$ satisfy a finite set of linear inequalities.

It can be proved (see $\overline{\mathrm{Mar}}$ ) that the closure $\overline{\mathcal{C}}$ of the minimal class $\mathcal{C}$ of $A$ is the union of the classes $\mathcal{C}^{\prime}$ over $\mathcal{C}\left(\mathcal{C} \prec \mathcal{C}^{\prime}\right)$, and we represent it by the convex set

$$
\overline{\mathcal{C}_{S}}=\{M \quad \mid S \subset S(M) \text { and } m(M)=1\},
$$

whose extremal edges are just the perfect forms in this set (whose number is finite).

From Voronoi theory we can deduce the following property of the ordered graph of minimal classes.

2.4 Theorem (cf. $\mathrm{Mar}]$ ). Let $\mathcal{C}$ and $\mathcal{C}^{\prime}$ be two minimal classes with respective ranks $t$ and $t^{\prime}$, such that $\mathcal{C} \prec \mathcal{C}^{\prime}$. Then there exists a finite sequence $\mathcal{C}_{0}=\mathcal{C} \prec$ $\mathcal{C}_{1} \prec \cdots \prec \mathcal{C}_{r}=\mathcal{C}^{\prime}$ for which $\mathcal{C}_{i}$ has rank of perfection $t+i$.

Proof. Let $A$ in $\mathcal{C}$ and $A^{\prime}$ in $\mathcal{C}^{\prime}$ be matrices scaled to minimum 1, with corresponding Voronoi domains $\mathcal{D}(A) \subset \mathcal{D}\left(A^{\prime}\right)$. There exists a hyperplane face $\mathcal{F}$ of $\mathcal{D}\left(A^{\prime}\right)$ containing $\mathcal{D}(A)$ and a matrix $A^{\prime \prime}$ such that $\mathcal{D}\left(A^{\prime \prime}\right)=\mathcal{F}$ (this is a basic idea of the Voronoi algorithm for quadratic forms: if $F$ is a nonzero vector orthogonal to $\mathcal{F}$ in the half-space containing $\mathcal{D}\left(A^{\prime}\right)$, then any matrix $A^{\prime \prime}=A^{\prime}+\epsilon F$ with $\epsilon>0$ small enough fits). Then the rank of perfection of the class $\mathcal{C}\left(A^{\prime \prime}\right)$ is just $t^{\prime}-1$. This proves the theorem inductively.

It follows that our algorithm, which involves the determination of all hyperplane faces of a given Voronoi domain, starting from all inequivalent perfect matrices, provides all minimal classes in a given dimension $n$. 
The stabilizer $\operatorname{Aut}(S)$ in $\mathrm{GL}_{\mathrm{n}}(\mathbb{Z})$ of a finite set $S \subset \mathbb{Z}^{n}$ is a finite group when $S$ spans $\mathbb{R}^{n}$. When $S=S(A)$ is the set of minimal vectors of a symmetric positive definite matrix $A, \operatorname{Aut}(S)$ stabilizes the convex $\mathcal{C}_{S}$ and its closure, and is an invariant for the minimal class $\mathcal{C}$ of $A$ up to conjugacy under $\mathrm{GL}_{\mathrm{n}}(\mathbb{Z})$. It will be referred to as the automorphism group of the class.

The automorphism group $\operatorname{Aut}(A)$ of any matrix $A$ in $\mathcal{C}_{S}$ is a subgroup of $\operatorname{Aut}(S)$. Actually, when the latter is finite, it can be realized via the usual arithmetic mean tool:

2.5 Proposition. Let $\mathcal{C}_{S}$ be a well-rounded minimal class, $A$ an element of $\mathcal{C}_{S}$ (resp. $\overline{\mathcal{C}_{S}}$ ), and

$$
M=\frac{1}{|\operatorname{Aut}(S)|} \sum_{U \in \operatorname{Aut}(S)}{ }^{t} U A U
$$

its arithmetic mean.

(1) $M \in \mathcal{C}_{S}\left(\right.$ resp. $\left.\overline{\mathcal{C}_{S}}\right)$.

(2) $\operatorname{Aut}(M)=\operatorname{Aut}(S)($ resp. $\operatorname{Aut}(M) \supset \operatorname{Aut}(S))$.

(3) $\operatorname{det}(M) \geq \operatorname{det}(A)$, and equality holds if and only if $M=A$ (hence $\operatorname{Aut}(A)=$ $\operatorname{Aut}(S)(\operatorname{resp} . \operatorname{Aut}(A) \supset \operatorname{Aut}(S)))$.

Proof. The first assertion is just the convexity of the class $\mathcal{C}_{S}$ and of its closure. The inclusion $\operatorname{Aut}(M) \supset \operatorname{Aut}(S)$ is trivial, and implies equality when $M$ lies in the class $\mathcal{C}_{S}$ itself, since its automorphism group is then a subgroup of $\operatorname{Aut}(S)$. The strict logarithmic concavity of the determinant (cf. [CS]) implies (3), as the equivalent matrices in the mean sum have the same determinant.

Again, from the strict logarithmic concavity of the determinant, it follows that the determinant assumes its maximum value in $\overline{\mathcal{C}_{S}}$ in a unique matrix. From 2.5 we obtain:

2.6 Corollary. The matrix $A \in \overline{\mathcal{C}_{S}}$ at which the determinant attains its maximum in $\overline{\mathcal{C}_{S}}$ is such that

$$
\operatorname{Aut}(A) \supset \operatorname{Aut}(S)
$$

(and equality holds when $A$ lies in $\mathcal{C}_{S}$ ).

2.7 Remark. Note that the number of forms integrally equivalent to $A$ in the set $\mathcal{C}_{S}$ is just the index of $\operatorname{Aut}(A)$ in $\operatorname{Aut}(S)$. Actually this convex set is not a fundamental domain of the class with respect to the integral equivalence unless the group $\operatorname{Aut}(A)$ is constant in the class.

2.8 Two more invariants. Let us introduce for the class $\mathcal{C}_{S}$ two new invariants useful for the computation of its automorphism group and its identification.

The first one comes from the characteristic determinants of order $n$ extracted from $S$ : for any subset $\left\{x_{1}, x_{2}, \ldots, x_{n}\right\} \subset S$, the absolute value of its determinant (a nonnegative integer bounded by the absolute constant $\gamma_{n}^{n / 2}$ ) is invariant under sign-changes and permutations of the $x_{i}$ 's. So we define the determinant spectrum of the class $\mathcal{C}$ to be the system of integers $\left(n_{0}, n_{1}, \ldots, n_{k}\right), k=\left[\gamma_{n}^{n / 2}\right]$, where $n_{i}$ is the number of subsets $\left\{ \pm x_{1}, \pm x_{2}, \ldots, \pm x_{n}\right\}$ of $S$ for which $\operatorname{det}\left(x_{1}, x_{2}, \ldots x_{n}\right)= \pm i$ (we have $\sum_{i} n_{i}=\left(\begin{array}{l}s \\ n\end{array}\right)$ ). the inequality $n_{1}>0$ means that the lattices of the class possess a basis of minimal vectors. Note that each $n_{i}$ is an increasing function on the set of classes. For instance the perfect class associated to the root lattice $\mathbb{A}_{n}$ is 
TABLE 1. Determinant spectrums of perfect forms in dimension 6

\begin{tabular}{|r|c|r|r|r|r|r|}
\hline form & $\mathbf{s}$ & \multicolumn{1}{c|}{$\mathbf{n}_{\mathbf{0}}$} & \multicolumn{1}{c|}{$\mathbf{n}_{\mathbf{1}}$} & \multicolumn{1}{|c|}{$\mathbf{n}_{\mathbf{2}}$} & \multicolumn{1}{|c|}{$\mathbf{n}_{\mathbf{3}}$} & $\mathbf{n}_{\mathbf{4}}$ \\
\hline$P_{6}^{1}$ & 36 & 1101072 & 798984 & 46656 & 1080 & 0 \\
$P_{6}^{2}$ & 27 & 165690 & 127224 & 3024 & 72 & 0 \\
$P_{6}^{3}$ & 30 & 365720 & 220800 & 7240 & 0 & 15 \\
$P_{6}^{4}$ & 22 & 45493 & 28830 & 288 & 2 & 0 \\
$P_{6}^{5}$ & 21 & 32984 & 21056 & 224 & 0 & 0 \\
$P_{6}^{6}$ & 21 & 33607 & 20501 & 156 & 0 & 0 \\
$P_{6}^{7}$ & 21 & 37457 & 16807 & 0 & 0 & 0 \\
\hline
\end{tabular}

characterized by $n_{i}=0$ if $i>1$ and $n_{1} \neq 0$ (actually, $n_{1}=(n+1)^{n-1}$, as proved by Roland Bacher using Cayley's formula for graphs). We also have computed this invariant for the senary perfect forms, confirming a result of Watson [W] (corrected by Martinet). In order of decreasing density, and with the terminology of [CS], we obtain Table 1.

A class is given by a subset $S$ of $\mathbb{Z}^{n}$, identified with a $n \times s$ integral matrix, defined up to permutation and sign-changes. The second invariant, suggested by Roland Bacher, is the integral $n \times n$ matrix

$$
T=S^{t} S=\sum_{x \in S} x^{t} x
$$

$T$ only depends on the set $S$, has the same rank as $S$, so it is positive definite for well-rounded classes.

2.9 Proposition (A.-M. Bergé). Let $\mathcal{C}_{S}$ be a well-rounded minimal class. We have the equality

$$
\operatorname{Aut}(S)=\operatorname{Aut}\left(T^{-1}\right) .
$$

Proof. We introduce the signed-permutation group $G_{n}$ of order $2^{n} \dot{n}$ !

$$
G_{n}=\left\{V \in \mathrm{GL}_{\mathrm{n}}(\mathbb{Z}) \mid{ }^{t} V V=I_{n}\right\} .
$$

The group of the class is just

$$
G=\operatorname{Aut}(S)=\left\{U \in \mathrm{GL}_{\mathrm{n}}(\mathbb{Z}) \mid \exists V \in G_{s}, U S=S V\right\}
$$

(the matrix $V$ corresponds to permutations and sign-changes). The inclusion

$$
\operatorname{Aut}(S) \subset \operatorname{Aut}\left(T^{-1}\right)=\left\{{ }^{t} U \mid U \in \operatorname{Aut}(T)\right\}
$$

is trivial. Conversely, if $U \in \operatorname{Aut}\left(T^{-1}\right)$, we have $S^{\prime}{ }^{t} S^{\prime}=S{ }^{t} S$ if $S^{\prime}=U S$. Let $A$ be an element of $\mathcal{C}_{S}$. We have

$$
\left\langle S^{\prime} S^{\prime}, A\right\rangle=\sum_{x^{\prime} \in S^{\prime}} x^{\prime} A^{t} x^{\prime}=\left\langle S^{t} S, A\right\rangle=\sum_{x \in S} x A^{t} x=s
$$

(using the fact that the vectors in $S$ are minimal vectors of norm 1 of $A$ ), so $S^{\prime}$ must be a permutation of $S$ up to sign-change and there exists $V$ in $G_{s}$ such that $U S=S^{\prime}=S V$. 


\section{Eutaxy}

Let us recall the definition of weak eutaxy ( $\underline{\mathrm{BM}})$ :

A symmetric positive definite matrix (or form) $A$ is said to be weakly eutactic if the inverse matrix $A^{-1}$ lies in the space spanned by the symmetric matrices $x^{t} x$, $x \in S(A)$ : there are real numbers $\rho_{x}$ (the eutaxy coefficients in [CS] such that

$$
A^{-1}=\sum_{x \in S(A)} \rho_{x} x^{t} x .
$$

By averaging, we can assume that $\rho_{x}$ is constant along the orbits under $\operatorname{Aut}(A)$.

Forms for which there exists a system of nonnegative eutaxy coefficients are called semi-eutactic in $\mathrm{CS}$; they are precisely those for which the inverse matrix $A^{-1}$ lies in the Voronoi domain of $A$. The form is said to be eutactic when there exists a system of strictly positive eutaxy coefficients.

Note that every perfect form is weakly eutactic.

We begin by two easy and well-known properties of weakly eutactic forms or matrices, which are useful for the classification of weakly eutactic forms. The first one implies that, if we are searching for weakly eutactic forms, we need only consider well-rounded classes (see $\S 2$ ). The second one asserts that among the $n$-dimensional weakly eutactic forms there are disconnected ones, arising from lower dimensions:

3.1 Proposition. (1) Let $Q$ be a weakly eutactic positive definite quadratic form. Then the set $S(Q)$ of its minimal vectors contains $n$ independent vectors.

(2) The orthogonal sum of two eutactic (resp. weakly eutactic) forms of dimensions $p$ and $p^{\prime}$ with the same minimum is a eutactic (resp. weakly eutactic) $p+p^{\prime}$-dimensional form.

Proof. (1) Let $A$ be the matrix of $Q$. A eutactic relation $A^{-1}=\sum_{x \in S(A)} \rho_{x} x^{t} x$ is just a decomposition of the adjoint form $y \mapsto{ }^{t} y A^{-1} y$ of $Q$ into a sum of squares of the linear forms $y \mapsto t y x, x \in S(A)$, whose rank is equal to $\operatorname{rank}(S(A))$. (Actually, the set of $x$ 's with $\rho_{x}>0$ in the above relation must span $\mathbb{R}^{n}$.)

(2) We have $S\left(Q+Q^{\prime}\right)=S(Q) \cup S\left(Q^{\prime}\right)$, and the adjoint of $Q+Q^{\prime}$ is equal to the orthogonal sum of the adjoints of $Q$ and $Q^{\prime}$.

Now we will investigate the connection between minimality of the Hermite invariant and eutaxy, making precise the characterization of weak eutaxy given in [BM] and providing a new proof of it.

3.2 Theorem. Let $\mathcal{C}$ be a minimal class of $n$-dimensional positive definite quadratic forms and $\overline{\mathcal{C}}=\cup_{\mathcal{C}^{\prime} \succ \mathcal{C}} \mathcal{C}^{\prime}$ its closure.

(1) The weakly eutactic forms in $\mathcal{C}$ (if any) are just those at which the Hermite invariant assumes its minimum in $\overline{\mathcal{C}}$.

(2) In each minimal class $\mathcal{C}$, there is at most one weakly eutactic form up to integral equivalence. More precisely, there is at most one weakly eutactic positive definite quadratic form with prescribed minimum and set of minimal vectors.

(3) Let $Q$ be a weakly eutactic form in $\mathcal{C}$ with eutaxy coefficients $\rho_{x}, x \in S(Q)$. Then any class obtained from $\mathcal{C}$ by removing minimal vectors of $Q$ with nonpositive coefficients $\rho_{x}$ has no weakly eutactic form.

(4) If the least dense of the weakly eutactic forms in $\overline{\mathcal{C}} \backslash \mathcal{C}$ is eutactic, then $\mathcal{C}$ contains weakly eutactic forms. 
For instance, a class of defect one is a Voronoi neighbouring path between two perfect forms. If these two perfect forms are extreme (hence eutactic), we conclude from (4) that the path contains a weakly eutactic form. This holds up to dimension 5. In dimension 6, Theorem 3.2 (4) applies in all cases, except for the neighbouring paths from the extreme sextic form $P_{6}^{1}$ (twice), $P_{6}^{2}, P_{6}^{3}$ to the sextic semi-eutactic perfect form $P_{6}^{4}$; actually, the class from $P_{6}^{2}$ to $P_{6}^{4}$ is unique without a weakly eutactic form: thus among the 18 classes with defect 1, 17 contain a weakly eutactic form.

Proof of Theorem 3.2. We work in the Euclidean space of the symmetric $n \times n$ matrices equipped with the inner product $\langle A, B\rangle=\operatorname{Tr}(A B)$. We normalize symmetric positive definite matrices to the same minimum $m$, thus we are looking for the maxima of the determinant. The differential of this function at $A$ is the linear form $F \mapsto \operatorname{det}(A)\left\langle A^{-1}, F\right\rangle$.

As usual the class $\mathcal{C}$ of $A$ will be represented by the polyhedron $\mathcal{C}_{S}$ (see Section 2), $(S=S(A))$ spanning an affine variety $A+Y$, where $Y$ is the orthogonal complement to the vector space $X$ spanned by the $x^{t} x, x \in S(A)$. Denoting by $\operatorname{det}_{\mid \overline{\mathcal{C}}}$ the restriction to $\overline{\mathcal{C}_{S}}$ of the determinant and using its strict logarithmic concavity, we have the following equivalences:

$$
\operatorname{det}(A)=\max \left(\operatorname{det}_{\mid \overline{\mathcal{C}}}\right) \Longleftrightarrow \operatorname{diff}_{A}\left(\operatorname{det}_{\mid \overline{\mathcal{C}}}\right)=0 \Longleftrightarrow \forall F \in Y,\left\langle A^{-1}, F\right\rangle=0 \Longleftrightarrow A^{-1} \in X,
$$

where the right-hand side condition is just the weak eutaxy of $A$.

So far we have proved (1) and (2) from concavity again.

From now on, we will assume that $A$ is a weakly eutactic symmetric positive definite matrix, and

$$
A^{-1}=\sum_{x \in S} \rho_{x} x^{t} x
$$

a relation of weak eutaxy.

Let $B \neq A$ be a symmetric positive definite matrix with minimum $m$ as stated before. We then have, for all $x \in S$,

$$
\left\langle x^{t} x, B\right\rangle=m+\epsilon_{x}
$$

with $\epsilon_{x} \geq 0$ and $\epsilon_{x}=0$ if and only if $x \in S(B)$. It follows that

$$
\left\langle A^{-1}, B\right\rangle=\sum_{x \in S} \rho_{x} m+\sum_{x \in S} \rho_{x} \epsilon_{x}=n+\sum_{x \in S} \rho_{x} \epsilon_{x} .
$$

We first assume

$$
S(B) \supset\left\{x \in S \mid \rho_{x}>0\right\} .
$$

The term $\sum_{x \in S} \rho_{x} \epsilon_{x}$ is then nonpositive, since $\epsilon_{x}$ vanishes for any $x \in S$ such that $\rho_{x}>0$. So we have

$$
\operatorname{Tr}\left(A^{-1} B\right) \leq n
$$

Now, by simultaneously diagonalizing $A$ and $B$ and applying the arithmetic-geometric mean inequality, we have

$$
\operatorname{det}\left(A^{-1} B\right)<\left(\frac{1}{n} \operatorname{Tr}\left(A^{-1} B\right)\right)^{n}
$$


(equality would imply that the matrix $A^{-1} B$ is scalar, and therefore, from the normalization, $A=B$ ). It follows that, under our hypothesis, $\operatorname{det}(B)<\operatorname{det}(A)$. We can now prove $(3)$. Let $\mathcal{C}^{\prime}$ be a minimal class obtained by removing from $S$ some $x$ with $\rho_{x} \leq 0$. Any form in $\mathcal{C}^{\prime}$ can be represented up to equivalence by a matrix $B \neq A$ satisfying

$$
S \supset S(B) \supset\left\{x \in S \mid \rho_{x}>0\right\},
$$

and therefore satisfies $\operatorname{det}(B)<\operatorname{det}(A)$. As $A$ lies in $\overline{\mathcal{C}^{\prime}}$ (from $S \supset S(B)$ ), we conclude from (1) that $B$ is not weakly eutactic.

We now assume

$$
\left\{x \in S \mid \rho_{x} \leq 0\right\} \subset S(B) \text { and } S \not \subset S(B) .
$$

The sum $\sum_{x \in S} \rho_{x} \epsilon_{x}$ is then positive (since $\epsilon_{x}=0$ if $\rho_{x} \leq 0$ and $\epsilon_{x}>0$ for some $x \in S)$, and so is the differential $F \mapsto \operatorname{Tr}\left(A^{-1} F\right)$ at $A$ of the determinant at $F=B-A$ :

$$
\left\langle A^{-1}, F\right\rangle=\left\langle A^{-1}, B\right\rangle-n=\sum_{x \in S \mid \rho_{x}>0} \rho_{x} \epsilon_{x} .
$$

Thus any matrix $M$ close enough to $A$ on the path from $A$ to $B$ satisfies $\operatorname{det}(M)>$ $\operatorname{det}(A)$. Now let $\mathcal{C}$ be as in (4), represented by $\mathcal{C}_{S}$, and let $A$ realize the maximum of the determinant in $\overline{\mathcal{C}_{S}}$. If $A$ were not in $\mathcal{C}_{S}$, it would be eutactic (from hypothesis (4)), and thus then for any $B \in \mathcal{C}_{S}$ the above condition (S) would hold for a convenient system of $\rho_{x}$, so $A$ would not be a maximum of the determinant in the path from $A$ to $B$ contained in $\overline{\mathcal{C}}$, a contradiction. This completes the proof of the theorem.

We can state again Corollary 2.6 in terms of eutaxy:

3.3 Corollary. The automorphism group of a weakly eutactic positive definite quadratic form is exactly the automorphism group of the class, i.e., the stabilizer Aut $(S)$ in $\mathrm{GL}_{\mathrm{n}}(\mathbb{Z})$ of the set $S$ of its minimal vectors.

We deduce from Section 2 that when searching for the weakly eutactic matrix in a class $\mathcal{C}_{S}$, we need only consider the matrices of $\mathcal{C}_{S}$ belonging to the linear variety $($ defined over $\mathbb{Q})$

$$
V^{\operatorname{Aut}(S)}=\left\{M \mid{ }^{t} U M U=M, U \in \operatorname{Aut}(S)\right\}
$$

of $n \times n$ real matrices fixed by $\operatorname{Aut}(S)$.

A weakly eutactic matrix is of course algebraic (i.e., has algebraic entries when conveniently scaled), since it is the unique solution in its class for the vanishing of the differential of the determinant. We can give some information about the field of definition.

3.4 Proposition. (1) The real conjugates of a weakly eutactic matrix $A$ (if any) are not positive definite.

(2) If the intersection $V^{\operatorname{Aut}(S)} \cap \overline{\mathcal{C}_{S}}$ is reduced to a single matrix $A$, then $A$ is rational, lies in $\mathcal{C}_{S}$, and is weakly eutactic.

(3) If this intersection is a segment, the weakly eutactic matrix which realizes the maximum of determinant in $\overline{\mathcal{C}_{S}}$ is defined over a totally real number field.

Proof. To prove (1), let $A^{\prime}$ be any real positive definite matrix in $\mathbb{R} \mathcal{C}_{S}$ where the differential of the determinant vanishes (for instance a conjugate of $A$ ), and let $\varepsilon>0$ be small enough for the matrices $A_{t}=t A+(1-t) A^{\prime}$ to be positive definite on the 
interval $I=[-\varepsilon, 1+\varepsilon]$. The logarithm of the determinant of $A_{t}$, a strictly concave function on $I$, has at least two extrema (at $t=0$ and $t=1$ ), a contradiction. For (2), apply Corollary 3.3 and note that $V^{\operatorname{Aut}(S)} \cap \mathcal{C}_{S}$ contains rational matrices (by $\operatorname{Aut}(S)$-averaging a rational matrix of the class). It only remains to establish assertion (3). $V^{\operatorname{Aut}(S)} \cap \overline{\mathcal{C}_{S}}$ can be parametrized as follows:

$$
A_{t}=B+t F, \quad t \in[a, b],
$$

with $B$ and $F$ rational and $B$ positive definite. The parameter $t_{0} \in[a, b]$ corresponding to the considered weakly eutactic matrix is a root of the derivative of the rational polynomial

$$
\operatorname{det}\left(A_{t}\right)=\operatorname{det}(B) \operatorname{det}\left(I_{n}+t B^{-1} F\right) .
$$

By simultaneously diagonalizing $B$ and $F$, one sees that the eigenvalues of the matrix $B^{-1} F$ are real. Let us denote by $\lambda_{1}, \ldots, \lambda_{p}$ the nonzero ones. We then have

$$
\operatorname{det}\left(A_{t}\right)=\operatorname{det}(B) \prod_{1 \leq i \leq p}\left(1+t \lambda_{i}\right) .
$$

Thus, the roots of this polynomial being all real, so are the roots of its derivative from which we conclude that $t_{0}$ is totally real (and with degree at most $\operatorname{rank}(F)-$ $1)$.

3.5 Remark. Up to dimension 5, all weakly eutactic matrices are defined over a totally real number field. However, examples of nontotally real matrices occur from dimension 6 onwards.

\section{Algorithms}

4.1 Algorithm for minimal classes. The algorithm consists in the enumeration of all inequivalent rank $(r-1)$-classes under a given rank $r$-class $\mathcal{C}_{S}$, starting from a perfect form $P$ and stopping when $\operatorname{rank}(S)=n$. The dual problem of construction of the hyperplane faces of the $r$-dimensional associated Voronoi domain $\mathcal{D}_{S}$ is a classical problem of linear programming, the heaviest step of the Voronoi algorithm. The use of the group of automorphisms of $S$ (or of a subgroup) is crucial to lowering the number of integrally equivalent faces produced: a new class is obtained by taking a certain subset $T \subset S$ away, and all classes corresponding to elements of the $\operatorname{Aut}(S)$-orbit of $T$ (up to sign-changes) are integrally equivalent. For instance, when $r=s$, the hyperplane faces are obtained by removing one vector, and there is a one-to-one correspondence between the $\operatorname{Aut}(S)$-orbits of vectors and $\operatorname{Aut}(S)$-orbits of the opposite faces. (Of course, two faces may be integrally but not $\operatorname{Aut}(S)$ equivalent.) An accurate description of the different types of faces in the case of dimension 5 is given in the next section.

We now describe a parametrization of the classes under $P$ conforming to our step-by-step descending path in the graph of minimal classes. We adapt to the Voronoi domain of $P$ the following more general linear programming:

Let $(V,\langle\rangle$,$) be a Euclidean space, \mathcal{D}$ the convex hull of a finite subset $D$ of $V, N$ its dimension, and let

$$
\mathcal{D}_{0}=\mathcal{D} \supset \mathcal{D}_{1} \supset \cdots \supset \mathcal{D}_{k-1} \supset \mathcal{D}_{k}
$$

be a descending chain of convex polyhedrons such that $\mathcal{D}_{i}$ is a hyperplane face of $\mathcal{D}_{i-1}$. We inductively construct a sequence $\mathcal{F}_{1}, \mathcal{F}_{2}, \cdots, \mathcal{F}_{i}, \cdots, \mathcal{F}_{k}$ of $(N-1)$-dimensional faces of the polyhedron $\mathcal{D}$ such that for $i=1,2, \cdots, k$, 
$\mathcal{D}_{i}=\mathcal{F}_{1} \cap \mathcal{F}_{2} \cap \cdots \cap \mathcal{F}_{i}$. Actually, we represent a face $\mathcal{F}$ of $\mathcal{D}$ by a so-called face-vector $F$, namely a vector orthogonal to the face such that $\langle F, v\rangle>0$ for all $v \in \mathcal{D} \backslash \mathcal{F}$. We then have to determine a sequence $\left(F_{1}, \cdots, F_{i}, \cdots, F_{k}\right)$ of facevectors of $\mathcal{D}$ such that, for $i=1,2, \cdots, k,\left\{F_{1}, F_{2}, \cdots, F_{i}\right\}$ is a base of the subspace $\mathcal{D}_{i}^{\perp}$ orthogonal to $\mathcal{D}_{i}$.

Algorithm. Let $D_{i}=D \cap \mathcal{D}_{i}$ be the subset of $D$ whose convex hull is $\mathcal{D}_{i}$, and define by induction

$$
F_{i}=\sum_{j=1}^{i-1} m_{i, j} F_{j}+f_{i} \quad(i=1, \cdots, k)
$$

where $f_{i} \in X_{i-1}$ is a face-vector for the face $\mathcal{D}_{i}$ of the convex set $\mathcal{D}_{i-1}$, and

$$
m_{i, j}=-\min _{v \in D_{j-1} \backslash D_{j}} \frac{\left\langle\sum_{h=j+1}^{i-1} m_{i, h} F_{h}+f_{i}, v\right\rangle}{\left\langle F_{j}, v\right\rangle} \quad(j=i-1, \cdots, 1) .
$$

Since we can avoid the general algorithm in dimension up to 5 (see section 5 ), we just sketch the proof. Let us assume that $F_{1}, \ldots, F_{i-1}$ is a suitable basis of $\mathcal{D}_{i-1}^{\perp}$ and let $F_{i}$ be defined as above: for all $v \in V$ we have

$$
\left\langle F_{i}, v\right\rangle=\sum_{j=1}^{i-1} m_{i, j}\left\langle F_{j}, v\right\rangle+\left\langle f_{i}, v\right\rangle
$$

We first note that, for $v \in \mathcal{D}_{i-1}$, all terms vanish except $\left\langle f_{i}, v\right\rangle$, which is (by construction) $>0$ on $\mathcal{D}_{i-1} \backslash \mathcal{D}_{i}$ and $=0$ on $\mathcal{D}_{i}$. So $F_{i} \in \mathcal{D}_{i}^{\perp} \backslash \mathcal{D}_{i-1}^{\perp}$ and $F_{1}, \ldots, F_{i}$ is a basis of $\mathcal{D}_{i}^{\perp}$.

It remains to check that $F_{i}^{\perp}$ is a face of $\mathcal{D}$. For $j \in\{1, \ldots, i-1\}$ and $v \in$ $\mathcal{D}_{j-1} \backslash \mathcal{D}_{j}$, we then have

$$
\left\langle F_{i}, v\right\rangle=\sum_{k=j}^{i-1} m_{i, k}\left\langle F_{k}, v\right\rangle+\left\langle f_{i}, v\right\rangle
$$

which is $\geq 0$ by choice of $m_{i, j}$ and vanishes for at least one vector $v_{j-1} \in \mathcal{D}_{j-1} \backslash \mathcal{D}_{j}$; so the hyperplane $F_{i}^{\perp}$ is spanned by $\mathcal{D}_{i}$ and $v_{0}, v_{1}, \ldots, v_{i-2} ;$ as $\left\langle F_{i}, v\right\rangle$ is $\geq 0$ on $\mathcal{D}$ we conclude that $F_{i}$ is a face-vector of $\mathcal{D}$.

4.2 Algorithm for the group of a class. As usual, the well-rounded class is given by a subset $S$ of $\mathbb{Z}^{n}$, identified with an $n \times s$ integral matrix of rank $n$. To compute the group $\operatorname{Aut}(S)$, we have just, by Proposition 2.9, to compute the group of the integral matrix $\operatorname{Aut}\left(S^{t} S\right)$. Note that if $A$ is the weakly eutactic matrix $A$ in the class (see subsection 4.3), we have also $\operatorname{Aut}(S)=\operatorname{Aut}(A)$ (Corollary 3.3). To compute the group of an integral definite positive matrix $A$ is a very classical problem (see [PS] for instance). To deal with matrices defined over a number field $K$, we readily adapted the former algorithm to formal computation. This algorithm is much less efficient than the classical one, so in the irrational case, we use the first matrix. 


\subsection{Algorithm for the weakly eutactic form. Let}

$$
\mathcal{C}_{S}=\left\{A_{t}=P+t_{1} F_{1}+\cdots+t_{k} F_{k} \quad t=\left(t_{1}, \cdots, t_{k}\right) \in \mathcal{T}\right\}
$$

be the parametrization of the class $\mathcal{C}_{S}$ derived from subsection 4.1, where $\mathcal{T}$ is a convex polyhedron spanning $\mathbb{R}^{k}$ (whose boundary is difficult to make precise - we generally only know that it contains the origin). The determinant function

$$
d\left(t_{1}, t_{2}, \cdots, t_{k}\right)=\operatorname{det}\left(A_{t}\right)
$$

is a polynomial with rational coefficients and degree at most $n$; it attains its maximum at the unique solution $\theta=\left(t_{1}, \cdots, t_{k}\right) \in \overline{\mathcal{T}}$ of the algebraic system

$$
\left\{\begin{array}{l}
\frac{\partial d}{\partial t_{1}}\left(t_{1}, \cdots, t_{k}\right)=0 \\
\frac{\partial d}{\partial t_{2}}\left(t_{1}, \cdots, t_{k}\right)=0 \\
\cdots \cdots \cdots, \cdots, \cdots, \\
\frac{\partial d}{\partial t_{k}}\left(t_{1}, \cdots, t_{k}\right)=0
\end{array}\right.
$$

whose direct exact resolution (using for instance the MAPLE function "solve") is becoming rather tedious for high values of $\mathrm{k}$. Moreover, it provides all solutions of (S) (as rational numbers or roots of polynomials) as well as some extra polynomials, among which it remains to select the correct one, namely the one leading to a matrix $A_{t}$ in the class (or its boundary). (Note that every solution of (S) corresponds to a symmetric matrix $A_{t}$ such that $\left\langle A_{t}, x^{t} x\right\rangle=1, x \in S$ and whose adjoint belongs to the span of the $x^{t} x, x \in S$.) The best candidate $t=\left(t_{1}, t_{2}, \cdots, t_{k}\right) \in \mathbb{R}^{k}$ is experimentally the closest to the origin, and to guarantee its accuracy it suffices to ascertain that the corresponding matrix $A_{t}$ has minimum 1 and is in our class by computing its minimal vectors. In the algebraic case, we use a close rational approximation: to make the check more rigorous, we could eliminate the other real solutions of $(\mathrm{S})$ by calculations of the signs of the principal determinants of the associate matrices (see Proposition 3.4), noting that the partial minors of order $k$ not only should be positive, but greater than $\gamma_{k}^{k}$.

Actually, to the exact resolution we prefered the classical gradient method which efficiently provides an approximate solution in $\mathcal{T}$ (if any). We look for the maximum of a convex function, and if this maximum exists in an open convex set, the algorithm converges to this solution. We search for a good enough approximation to obtain when necessary (by the "algdep" PARI function based on LLL algorithm) an irreducible polynomial $f$ defining the number field $K=\mathbb{Q}(\theta)$. It remains to check the accuracy of this solution $\theta$ by formal computation of a basis of the systems of eutactic coefficients in $K$ (this is the classical determination of the kernel of a matrix with entries integral or polynomial modulo $f$ ).

When the gradient algorithm gives an erratic solution, we conclude that the determinant attains its maximum on the least dense of the weakly eutactic forms on the boundary of the class.

4.4 Identification of a class. In the above sections, we attached to a minimal class, represented by the set $\mathcal{C}_{S}$ of matrices with minimum 1 and set of minimal vectors $S$, some invariants: the number $2 s$ of minimal vectors, the rank of perfection, the determinant spectrum, the order of its automorphism group, the weakly eutactic matrix in its closure where the determinant attains its maximum, with its number 
field and density, and finally the invariants of the integral matrix $T=S^{t} S$. If they coincide for two classes $\mathcal{C}_{S_{1}}$ and $\mathcal{C}_{S_{2}}$, we must look for a matrix $U$ in $\operatorname{GL}_{\mathrm{n}}(\mathbb{Z})$ such that $U S_{1}=S_{2} V$, where $V$ is a permutation and sign-changes $s \times s$ matrix: this is with minor modifications the problem alluded to in subsection 4.2 , but here we stop with the first convenient matrix $U$ (if any). Note that if we choose to compare two weakly eutactic matrices, we must be careful with formal calculations in the algebraic case: if $A$ and $B$ are two matrices with entries in two isomorphic number fields defined by two distinct polynomials $f$ and $g$, we first have to find all automorphisms $\sigma$ from $\mathbb{Q}(\alpha)$ to $\mathbb{Q}(\beta)$ (where $\alpha$ and $\beta$ are roots of $f$ and $g$ ); for each $\sigma$, we obtain, by substituting $\beta$ by $\sigma(\alpha)$, two matrices over the same field $\mathbb{Q}(\alpha)$, and we look for integral equivalence between them in the usual method. Of course, we know from Proposition 3.4 (1) that there is at most one solution $\sigma$.

\section{QUINTIC FORMS}

5.1 Perfect Voronoi domains in dimension 5. We used here well-known results on low-dimensional forms (see [Mar], chap. VII, §7). The notation is that of [CS1].

We start the algorithm with the 3 perfect forms in dimension 5: $A_{5}, A_{5}^{3}$ and $D_{5}$. They are obtained by the classical Voronoi algorithm, beginning with

$$
A_{5}=\left(\sum_{1 \leq i \leq 5} x_{i}\right)^{2}+\sum_{1 \leq i \leq 5} x_{i}^{2} .
$$

The automorphism groups of the forms $A_{5}$ and $A_{5}^{3}$ act transitively on their sets of minimal vectors, so they have only (up to integral equivalence) one neighbour, which is

$$
D_{5}=A_{5}-2 x_{1} x_{2}
$$

The form $D_{5}$ has 20 pairs $( \pm v)$ of minimal vectors. Rejecting one of each pair and writing the others in a convenient order, we obtain the matrix

$S\left(D_{5}\right)=\left(\begin{array}{rrrrrrrrrrrrrrrrrrrr}1 & 0 & 0 & 0 & 0 & 1 & 1 & 1 & 0 & 0 & 0 & 0 & 0 & 0 & 1 & 1 & 1 & 1 & 1 & 1 \\ 0 & 1 & 0 & 0 & 0 & 0 & 0 & 0 & 1 & 1 & 1 & 0 & 0 & 0 & 1 & 1 & 1 & 1 & 1 & 1 \\ 0 & 0 & 1 & 0 & 0 & -1 & 0 & 0 & -1 & 0 & 0 & 1 & 1 & 0 & -1 & 0 & 0 & -1 & -1 & 0 \\ 0 & 0 & 0 & 1 & 0 & 0 & -1 & 0 & 0 & -1 & 0 & -1 & 0 & 1 & 0 & -1 & 0 & -1 & 0 & -1 \\ 0 & 0 & 0 & 0 & 1 & 0 & 0 & -1 & 0 & 0 & -1 & 0 & -1 & -1 & 0 & 0 & -1 & 0 & -1 & -1\end{array}\right)$.

So all classes with rank up to 14 can be obtained from $D_{5}$. The 400 faces of the Voronoi domain of $D_{5}$ are divided into 4 orbits leading to forms integrally equivalent to $A_{5}, D_{5}^{\prime} \sim D_{5}, A_{5}^{3}, E_{5} \sim D_{5}$, respectively. More precisely we have

$$
\begin{gathered}
A_{5}=D_{5}+2 x_{1} x_{2}, \\
D_{5}^{\prime}=D_{5}+2\left(x_{1} x_{2}-x_{3} x_{4}\right), \\
A_{5}^{3}=D_{5}+\left(x_{1} x_{2}-x_{3} x_{4}-x_{3} x_{5}-x_{4} x_{5}\right), \\
E_{5}=D_{5}-2 x_{1} x_{3} .
\end{gathered}
$$

When going from $D_{5}$ to one of these neighbours, we remove from $S\left(D_{5}\right) 6$ vectors except in the last case - let us call it special - where we take off 4 vectors, keeping the 12 minimal vectors spanning the $D_{4}$ section $x_{3}=0$ of $D_{5}$. The four corresponding inequivalent classes of rank 14 contain a eutactic form

$$
D_{5}+\frac{3}{2} x_{1} x_{2}
$$




$$
\begin{gathered}
D_{5}+\left(x_{1} x_{2}-x_{3} x_{4}\right), \\
D_{5}+\frac{5-\sqrt{10}}{5}\left(x_{1} x_{2}-x_{3} x_{4}-x_{3} x_{5}-x_{4} x_{5}\right), \\
D_{5}-x_{1} x_{3} .
\end{gathered}
$$

Note that the last eutactic form has $s=16>15=n(n+1) / 2$ pairs of minimal vectors, and is not extreme. This is the minimal negative answer to a question by Coxeter: Is every eutactic form with $s \geq n(n+1) / 2$ extreme? (see [Ba]). Its class is the only nonperfect one with $s \geq 16$.

Now, to obtain the complete graph of minimal classes in dimension 5 , we can restrict ourselves to classes under the perfect class of $D_{5}$ and have to distinguish two kinds of classes. In those for which $r=s$ are easy to compute, we remove from the set $S(C)$ one minimal vector, up to the isometry group of the class (or the group of an element of the class). Those for which $s>r$ are a little bit more complicated to deal with; they belong to a class we shall call "special". Actually, they satisfy $s=r+2$, for they correspond to lattices having a section similar to the $D_{4}$-lattice, for which $s=12$ and $r=10$. Note that this section is unique: a lattice with two sections $L_{1}$ and $L_{2}$ isometric to $\mathbb{D}_{4}$ must have at least $18=$ $12+12-6$ pairs of minimal vectors (the 3 -dimensional lattice $L_{1} \cap L_{2}$ having at most 6 minimal vectors). The subset of the 12 minimal vectors of the section $D_{4}$ is therefore invariant under the automorphism group of the class. In order to obtain the classes under a special class $\mathcal{C}_{S}$, we remove from the set $S$ either one minimal vector out of the $D_{4}$-section (producing a special class), or 3 minimal vectors in the $D_{4}$-section spanning a hexagonal plane or having mutual product 1 (corresponding to the two orbits of faces of the Voronoi domain of $D_{4}$ ). Taking into account the action of the group of the class on these subsets (only one orbit in the special case), we obtain a unique sequence of well-rounded special classes

$$
b_{11} \prec l_{12} \prec i_{13} \prec d_{14} \prec \text { class }\left(D_{5}\right)
$$

(the class $b_{11}$ has $A_{1} \perp D_{4}$ as a eutactic form, so its group is $C_{2} \times \operatorname{Aut}\left(D_{4}\right)$, with 2304 elements, the biggest one after $\left.\operatorname{Aut}\left(D_{5}\right)=\operatorname{Aut}\left(D_{5}^{*}\right)=\operatorname{Aut}\left(A_{1}^{5}\right)\right)$, and only two nonspecial classes $c_{13}$ and $g_{13}$ which could not be produced from nonspecial classes.

5.2 Results. Our algorithm for minimal classes was implemented on a Sun Sparc station under UNIX system environment. The computation times are very short, except for the determination of the field of definition of the eutactic form when a high degree is involved.

We conclude this paper with Table 3 giving the list of the well-rounded minimal classes in dimension 5 and their invariants, and Table 4 displaying the matrices of the rational weakly eutactic forms. The algebraic matrices and the automorphism groups can be requested from the author.

Let us give a few statistics and comments on our results.

There are 136 minimal classes in dimension 5, and no coincidence of their invariants. Among these classes, only 127 have a weakly eutactic form, among which 118 are eutactic.

Let us recall that there are, respectively, 1, 2, 5, 18 classes in dimension 1, 2, 3,4 , all of them with eutactic forms except two in dimension 4 (one with a semieutactic form and, as foreseen from Theorem 3.2 (3), a class below without any 
TABLE 2. Statistics on eutaxy in dimension 5

\begin{tabular}{|c|c|c|c|c|c|c|c|c|c|c|c|}
\hline & & \multicolumn{4}{|c|}{ Weakly eutactic form } & \multicolumn{6}{|c|}{ Degree of number field } \\
\hline rk & $\# \mathbf{c l}$ & e & se & we & no & 1 & 2 & 3 & 4 & 5 & 9 \\
\hline 15 & 3 & 3 & & & & 3 & & & & & \\
\hline 14 & 4 & 4 & & & & 3 & 1 & & & & \\
\hline 13 & 9 & 9 & & & & 5 & 3 & 1 & & & \\
\hline 12 & 16 & 15 & 1 & & & 8 & 4 & 3 & 1 & & \\
\hline 11 & 23 & 19 & 2 & 1 & 1 & 9 & 9 & 2 & & 1 & 1 \\
\hline 10 & 25 & 20 & & 2 & 3 & 9 & 10 & 2 & 1 & & \\
\hline 9 & 23 & 19 & 1 & 1 & 2 & 11 & 7 & 1 & 1 & 1 & \\
\hline 8 & 16 & 13 & 1 & & 2 & 7 & 6 & 1 & & & \\
\hline 7 & 10 & 9 & & & 1 & 7 & 2 & & & & \\
\hline 6 & 5 & 5 & & & & 5 & & & & & \\
\hline 5 & 2 & 2 & & & & 2 & & & & & \\
\hline Total & 136 & 118 & 5 & 4 & 9 & 69 & 42 & 10 & 3 & 2 & 1 \\
\hline
\end{tabular}

weakly eutactic form). In dimension 5 , the ratio of classes with eutactic forms is nearly the same, but negative eutaxy coefficients appear here in ranks 9, 10, and 11.

From Proposition 3.1 we know that our classification yields the classification in lower dimensions. Twenty disconnected weakly eutactic forms (of course eutactic indeed except one) appear in ranks up to 11 (the maximal rank in dimension 4 is 10). Their classes are the least dense in their rank.

Among the 127 weakly eutactic forms, only 69 are rational, the others are defined over totally real number fields with degrees up to nine. Note that the nine weakly eutactic noneutactic forms except one $\left(t_{11}\right.$ which is defined over the field $\left.\mathbb{Q}(\sqrt{13})\right)$ are rational. To save space, we will not describe the field of definition of weakly eutactic forms when the degree is greater than 2 . We will content ourselves with the single example of the field $K$ of degree 9 . It has discriminant $2^{4} \cdot 3^{2} \cdot 17^{2}$. $81037273 \cdot 3535337303$, and can be defined by the polynomial

$$
\begin{gathered}
x^{9}-2 x^{8}-150 x^{7}+174 x^{6}+5763 x^{5}-3564 x^{4} \\
-82450 x^{3}+18548 x^{2}+387552 x+18432 .
\end{gathered}
$$

(The computation of the corresponding eutactic form required an accuracy of about 450 digits. For the 5 degree polynomial of the class $w_{11}$ we needed about 100 digits, and for all other classes, less than 50 digits.) Table 2 summarizes these results.

When looking at the index $n_{1}$ of the determinant spectrums in Table 3 below, we see that each well-rounded class except one (the class $a_{5}$ of the eutactic lattice $D_{5}^{*}$ dual of $D_{5}$ ) has a basis of minimal vectors. More precisely we have (see [Mar]):

Proposition. Let $A$ be a positive definite quadratic form in dimension less than or equal to five, such that $S(A)$ contains $n$ independent minimal vectors. Then the sublattice of $\mathbb{Z}^{n}$ generated by $S(A)$ in $\mathbb{Z}^{n}$ is equal to $\mathbb{Z}^{n}$ except for the class of $D_{5}^{*}$ where the index is 2 and $s=5$. When the index is one, we can extract from $S(A)$ a basis of $\mathbb{Z}^{n}$. 
5.3 Ash's formula. Let us recall Ash's formula for $n$-dimensional eutactic forms:

$$
\sum_{A} \frac{(-1)^{i(A)}}{\left|\operatorname{Aut}^{+}(A)\right|}=\chi\left(\mathrm{SL}_{\mathrm{n}}(\mathbb{Z})\right),
$$

where $A$ ranges over all integrally inequivalent eutactic forms in dimension $n$. Here $i$ is the defect of perfection of the form and $\chi$ is the Euler-Poincaré characteristic of a group (note that $\chi\left(\mathrm{SL}_{n}(\mathbb{Z})\right)=0$ for $n \geq 3$ ). The sign + means that only direct equivalence is considered, but when the dimension is odd and greater than or equal to 3, we may consider the full group $\operatorname{Aut}(A)$ as defined in Section 2. Our calculations of automorphism groups (see Table 3) allow us to check Ash's formula for dimension 5, i.e., the vanishing of an alternating sum of 118 terms:

$$
\begin{gathered}
\frac{1}{1440}+\frac{1}{3840}+\frac{1}{1440} \\
-\left(\frac{1}{96}+\frac{1}{32}+\frac{1}{96}+\frac{1}{96}\right) \\
+\left(\frac{1}{24}+\frac{1}{32}+\frac{1}{32}+\frac{1}{8}+\frac{1}{8}+\frac{1}{32}+\frac{1}{72}+\frac{1}{24}+\frac{1}{72}\right) \\
-\left(\frac{1}{48}+\frac{1}{72}+\frac{1}{8}+\frac{1}{8}+\frac{1}{24}+\frac{1}{96}+\frac{1}{32}+\frac{1}{4}+\frac{1}{8}+\frac{1}{4}+\frac{1}{192}+\frac{1}{8}+\frac{1}{8}+\frac{1}{24}+\frac{1}{72}\right) \\
+\left(\frac{1}{480}+\frac{1}{2304}+\frac{1}{8}+\frac{1}{32}+\frac{1}{48} \frac{1}{4}+\frac{1}{24}+\frac{1}{16}+\frac{1}{8}+\frac{1}{8}+\frac{1}{16}+\frac{1}{8}+\frac{1}{48}+\frac{1}{4}+\frac{1}{4}+\frac{1}{20}+\frac{1}{4}+\frac{1}{96}+\frac{1}{8}\right) \\
-\left(\frac{1}{48}+\frac{1}{288}+\frac{1}{64}+\frac{1}{16}+\frac{1}{16}+\frac{1}{8}+\frac{1}{8}+\frac{1}{8}+\frac{1}{20}+\frac{1}{32}+\frac{1}{4}+\frac{1}{24}+\frac{1}{24}+\frac{1}{20}+\frac{1}{8}+\frac{1}{8}+\frac{1}{4}+\frac{1}{4}+\frac{1}{16}+\frac{1}{1440}\right) \\
+\left(\frac{1}{32}+\frac{1}{576}+\frac{1}{32}+\frac{1}{96}+\frac{1}{16}+\frac{1}{48}+\frac{1}{32}+\frac{1}{8}+\frac{1}{16}+\frac{1}{8}+\frac{1}{8}+\frac{1}{24}+\frac{1}{144}+\frac{1}{96}+\frac{1}{8}+\frac{1}{64}+\frac{1}{24}+\frac{1}{8}+\frac{1}{8}\right) \\
-\left(\frac{1}{384}+\frac{1}{192}+\frac{1}{32}+\frac{1}{32}+\frac{1}{96}+\frac{1}{32}+\frac{1}{24}+\frac{1}{48}+\frac{1}{16}+\frac{1}{64}+\frac{1}{24}+\frac{1}{16}+\frac{1}{24}\right) \\
+\left(\frac{1}{128}+\frac{1}{576}+\frac{1}{576}+\frac{1}{48}+\frac{1}{96}+\frac{1}{144}+\frac{1}{96}+\frac{1}{96}+\frac{1}{48}\right) \\
-\left(\frac{1}{576}+\frac{1}{384}+\frac{1}{480}+\frac{1}{1440}+\frac{1}{240}\right) \\
+\left(\frac{1}{3840}+\frac{1}{3840}\right) \\
=0 .
\end{gathered}
$$

Remark. Theorem 3.2 states that to any class $\mathcal{C}$ without a weakly eutactic form corresponds a class $\mathcal{C}^{\prime} \succ \mathcal{C}$ with a weakly eutactic form which is not eutactic (namely the class of the less dense form in $\overline{\mathcal{C}}$ ). In the five-dimensional case, this correspondence happens to be one-to-one, with consecutive defects and the same automorphism groups. Thus from the Ash formula we can deduce a "mass formula" for the five-dimensional well-rounded minimal classes (instead of eutactic forms)

$$
\sum_{\mathcal{C}} \frac{(-1)^{i(\mathcal{C})}}{|\operatorname{Aut}(\mathcal{C})|}=\chi\left(\mathrm{SL}_{\mathrm{n}}(\mathbb{Z})\right)
$$

Actually, a general proof of this formula has just been given to me by Christophe Bavard $([\mathrm{Bv}])$. In this second formula for $n=5$ we have 18 more terms $-\frac{1}{32}+\left(\frac{1}{64}+\right.$ $\left.\frac{1}{16}+\frac{1}{32}\right)-\left(\frac{1}{64}+\frac{1}{64}+\frac{1}{16}+\frac{1}{96}\right)+\left(\frac{1}{32}+\frac{1}{768}+\frac{1}{64}+\frac{1}{96}\right)-\left(\frac{1}{192}+\frac{1}{32}+\frac{1}{768}\right)+\frac{1}{192}=0$. 
TABLE 3. The index in our notation for the classes is the rank of perfection, and the classes of the same rank are ordered in ascending order of the minimal Hermite invariant $\gamma$ in the class (or its boundary). In the fourth column we give the order $g$ of the automorphism group of the class, and in the fifth the determinant spectrum $\left[n_{0}, n_{1}, n_{2}\right]$ of the class. In the sixth column we specify the existence of a weakly eutactic form in the class ("e" means eutactic, "se" semi-eutactic, and "we" the other types), and the name of remarkable or disconnected forms (in the last case, a notation like $\mathrm{E}\left(a_{8}^{4}\right)$ refers to the weakly eutactic form in the 4-dimensional class labelled $a_{8}$ in $[\mathrm{BM}]$ ). If there is no weakly eutactic form in the class, we refer to the one in the boundary at which the minimum of density is attained. In the last column, we give the number field over which the corresponding eutactic form is defined, or only its degree when it is greater than 2 .

\begin{tabular}{|r|r|c|r|r|l|r|}
\hline class & s & $\gamma$ & \multicolumn{1}{|c|}{ g } & \multicolumn{1}{c|}{ Spect } & Eut & \multicolumn{1}{|c|}{ Field } \\
\hline$a_{15}$ & 15 & 1.39765 & 1440 & {$[1707,1296,0]$} & $\mathrm{e}\left(A_{5}\right)$ & $\mathbb{Q}$ \\
$b_{15}$ & 15 & 1.44596 & 1440 & {$[1485,1512,6]$} & $\mathrm{e}\left(A_{5}^{3}\right)$ & $\mathbb{Q}$ \\
$c_{15}$ & 20 & 1.51571 & 3840 & {$[7792,7552,160]$} & $\mathrm{e}\left(D_{5}\right)$ & $\mathbb{Q}$ \\
\hline$a_{14}$ & 14 & 1.38629 & 96 & {$[1138,864,0]$} & $\mathrm{e}$ & $\mathbb{Q}$ \\
$b_{14}$ & 14 & 1.39765 & 32 & {$[1048,952,2]$} & $\mathrm{e}$ & $\mathbb{Q}$ \\
$c_{14}$ & 14 & 1.43378 & 96 & {$[990,1008,4]$} & $\mathrm{e}$ & $\mathbb{Q}(\sqrt{10})$ \\
$d_{14}$ & 16 & 1.44955 & 96 & {$[2364,1986,18]$} & $\mathrm{e}$ & $\mathbb{Q}$ \\
\hline$a_{13}$ & 13 & 1.36851 & 24 & {$[747,540,0]$} & $\mathrm{e}$ & $\mathbb{Q}$ \\
$b_{13}$ & 13 & 1.37737 & 32 & {$[711,576,0]$} & $\mathrm{e}$ & $\mathbb{Q}(\sqrt{172})$ \\
$c_{13}$ & 13 & 1.39190 & 32 & {$[702,584,1]$} & $\mathrm{e}$ & $\mathbb{Q}$ \\
$d_{13}$ & 13 & 1.39958 & 8 & {$[674,612,1]$} & $\mathrm{e}$ & $\mathbb{Q}(\sqrt{33})$ \\
$e_{13}$ & 13 & 1.40816 & 8 & {$[669,616,2]$} & $\mathrm{e}$ & $\mathrm{deg} 3$ \\
$f_{13}$ & 13 & 1.41229 & 32 & {$[653,632,2]$} & $\mathrm{e}$ & $\mathbb{Q}$ \\
$g_{13}$ & 13 & 1.41933 & 72 & {$[648,636,3]$} & $\mathrm{e}$ & $\mathbb{Q}$ \\
$h_{13}$ & 13 & 1.42376 & 24 & {$[624,660,3]$} & $\mathrm{e}$ & $\mathbb{Q}(\sqrt{57})$ \\
$i_{13}$ & 15 & 1.43096 & 72 & {$[1716,1275,12]$} & $\mathrm{e}$ & $\mathbb{Q}$ \\
\hline$a_{12}$ & 12 & 1.33656 & 48 & {$[492,300,0]$} & $\mathrm{e}$ & $\mathbb{Q}$ \\
$b_{12}$ & 12 & 1.35096 & 72 & {$[468,324,0]$} & $\mathrm{e}$ & $\mathbb{Q}$ \\
$c_{12}$ & 12 & 1.35096 & 8 & {$[456,336,0]$} & $\mathrm{e}$ & $\mathbb{Q}$ \\
$d_{12}$ & 12 & 1.36334 & 8 & {$[432,360,0]$} & $\mathrm{e}$ & $\operatorname{deg} 3$ \\
$e_{12}$ & 12 & 1.36511 & 24 & {$[429,363,0]$} & $\mathrm{e}$ & $\mathbb{Q}$ \\
$f_{12}$ & 12 & 1.36979 & 96 & {$[408,384,0]$} & $\mathrm{e}$ & $\mathbb{Q}$ \\
$g_{12}$ & 12 & 1.37646 & 32 & {$[400,392,0]$} & $\mathrm{e}$ & $\mathbb{Q}(\sqrt{10})$ \\
$h_{12}$ & 12 & 1.38353 & 4 & {$[424,367,1]$} & $\mathrm{e}$ & $\mathbb{Q}(\sqrt{385})$ \\
$i_{12}$ & 12 & 1.38936 & 8 & {$[411,380,1]$} & $\mathrm{e}$ & $\mathbb{Q}(\sqrt{145})$ \\
$j_{12}$ & 12 & 1.39206 & 4 & {$[400,391,1]$} & $\mathrm{e}$ & $\mathrm{deg} 3$ \\
$k_{12}$ & 12 & 1.39765 & 32 & {$[414,376,2]$} & $\mathrm{se}$ & $\mathbb{Q}$ \\
$l_{12}$ & 14 & 1.39765 & 192 & {$[1267,728,7]$} & $\mathrm{e}$ & $\mathbb{Q}$ \\
$m_{12}$ & 12 & 1.40248 & 8 & {$[402,388,2]$} & $\mathrm{e}$ & $\mathrm{deg} 4$ \\
$n_{12}$ & 12 & 1.40680 & 8 & {$[382,408,2]$} & $\mathrm{e}$ & $\mathbb{Q}(\sqrt{73})$ \\
$o_{12}$ & 12 & 1.41377 & 24 & {$[366,424,2]$} & $\mathrm{e}$ & $\mathrm{deg} 3$ \\
$p_{12}$ & 12 & 1.41714 & 72 & {$[369,420,3]$} & $\mathrm{e}$ & $\mathbb{Q}$ \\
\hline
\end{tabular}


Table 3 (continued)

\begin{tabular}{|c|c|c|c|c|c|c|}
\hline class & $\mathbf{s}$ & $\gamma$ & $\mathrm{g}$ & Spect & Eut & Field \\
\hline$a_{11}$ & 11 & 1.26191 & 480 & {$[337,125,0]$} & e $\quad\left(A_{4} \perp A_{1}\right)$ & $\mathbb{Q}$ \\
\hline$b_{11}$ & 13 & 1.31951 & 2304 & {$[972,312,3]$} & $\left(D_{4} \perp A_{1}\right)$ & $\mathbb{Q}$ \\
\hline$c_{11}$ & 11 & 1.31951 & 8 & {$[277,185,0]$} & $\mathrm{e}$ & $\mathbb{Q}$ \\
\hline$d_{11}$ & 11 & 1.31951 & 64 & {$[270,192,0]$} & se & $\mathbb{Q}$ \\
\hline$e_{11}$ & 11 & 1.31951 & 16 & {$[282,180,0]$} & se & $\mathbb{Q}$ \\
\hline$f_{11}$ & 11 & 1.33333 & 32 & {$[264,198,0]$} & $\mathrm{e}$ & $\mathbb{Q}$ \\
\hline$g_{11}$ & 11 & 1.33624 & 48 & {$[262,200,0]$} & $\mathrm{e}$ & $\mathbb{Q}(\sqrt{7})$ \\
\hline$h_{11}$ & 11 & 1.33932 & 4 & {$[253,209,0]$} & $\mathrm{e}$ & $\mathbb{Q}(\sqrt{3})$ \\
\hline$i_{11}$ & 11 & 1.34843 & 24 & {$[246,216,0]$} & $\mathrm{e}$ & $\mathbb{Q}(\sqrt{73})$ \\
\hline$j_{11}$ & 11 & 1.34843 & 16 & {$[238,224,0]$} & $\mathrm{e}$ & $\mathbb{Q}(\sqrt{73})$ \\
\hline$k_{11}$ & 11 & 1.35481 & 8 & {$[237,225,0]$} & $\mathrm{e}$ & $\mathbb{Q}(\sqrt{33})$ \\
\hline$l_{11}$ & 11 & 1.35686 & 8 & {$[231,231,0]$} & $\mathrm{e}$ & $\operatorname{deg} 3$ \\
\hline$m_{11}$ & 11 & 1.36845 & 16 & {$[255,206,1]$} & $\mathrm{e}$ & $\mathbb{Q}(\sqrt{97})$ \\
\hline$n_{11}$ & 11 & 1.37013 & 8 & {$[245,216,1]$} & $\mathrm{e}$ & $\mathbb{Q}(\sqrt{2})$ \\
\hline$o_{11}$ & 11 & 1.37152 & 48 & {$[249,212,1]$} & $\mathrm{e}$ & $\mathbb{Q}(\sqrt{145})$ \\
\hline$p_{11}$ & 11 & 1.37630 & 4 & {$[235,226,1]$} & e & $\mathbb{Q}$ \\
\hline$q_{11}$ & 11 & 1.37906 & 4 & {$[231,230,1]$} & e & $\operatorname{deg} 3$ \\
\hline$r_{11}$ & 11 & 1.38528 & 20 & {$[215,246,1]$} & $\mathrm{e}$ & $\mathbb{Q}$ \\
\hline$s_{11}$ & 11 & 1.38570 & 4 & {$[219,242,1]$} & $\mathrm{e}$ & $\operatorname{deg} 9$ \\
\hline$t_{11}$ & 11 & 1.39466 & 16 & {$[228,232,2]$} & we & $\mathbb{Q}(\sqrt{13})$ \\
\hline$u_{11}$ & 11 & 1.39765 & 32 & {$[212,248,2]$} & {$\left[\begin{array}{ll}k_{12} & \mathrm{se}\end{array}\right]$} & \\
\hline$v_{11}$ & 11 & 1.40206 & 96 & {$[195,266,1]$} & $\mathrm{e}$ & $\mathbb{Q}$ \\
\hline$w_{11}$ & 11 & 1.40214 & 8 & {$[204,256,2]$} & $\mathrm{e}$ & $\operatorname{deg} 5$ \\
\hline$a_{10}$ & 10 & 1.24573 & 48 & {$[177$,} & e $(\mathrm{E}(b)$ & $\mathbb{Q}$ \\
\hline$b_{10}$ & 10 & 1.25878 & 288 & {$[171,81,0]$} & e $\left(\mathrm{E}\left(a_{9}^{4}\right)\right.$ & $\mathbb{Q}$ \\
\hline$c_{10}$ & 10 & 1.28878 & 64 & {$[152,100,0]$} & e & $\mathbb{Q}$ \\
\hline$d_{10}$ & 10 & 1.28878 & 16 & {$[153,99,0]$} & $\mathrm{e}$ & $\mathbb{Q}$ \\
\hline$e_{10}$ & 10 & 1.28878 & 16 & {$[148,104,0]$} & e & $\mathbb{Q}$ \\
\hline$f_{10}$ & 10 & 1.28878 & 64 & {$[156,96,0]$} & we & $\mathbb{Q}$ \\
\hline$g_{10}$ & 10 & 1.30813 & 8 & {$[141,111,0]$} & $\mathrm{e}$ & $\mathbb{Q}(\sqrt{3})$ \\
\hline$h_{10}$ & 10 & 1.30813 & 8 & {$[138,114,0]$} & 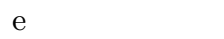 & $\mathbb{Q}(\sqrt{3})$ \\
\hline$i_{10}$ & 10 & 1.31668 & 8 & {$[137,115,0]$} & $\mathrm{e}$ & $\mathbb{Q}(\sqrt{13})$ \\
\hline$j_{10}$ & 10 & $1.31 \mathrm{~s}$ & 64 & {$[124,128,0]$} & e] & \\
\hline$k_{10}$ & 10 & 1.31951 & 16 & {$[132,120,0]$} & $e_{11}$ & \\
\hline$l_{10}$ & 10 & 1.31951 & 20 & {$[131,121,0]$} & $\mathrm{e}$ & $\mathbb{Q}(\sqrt{5})$ \\
\hline$m_{10}$ & 10 & 1.33206 & 32 & {$[126,126,0]$} & $\mathrm{e}$ & $\mathbb{Q}(\sqrt{19})$ \\
\hline$n_{10}$ & 10 & & 4 & {$[122,130,0]$} & e & $\operatorname{deg} 3$ \\
\hline$o_{10}$ & 10 & 1.34216 & 24 & {$[117,135,0]$} & $\mathrm{e}$ & $\mathbb{Q}$ \\
\hline$p_{10}$ & 10 & 1.34416 & 24 & {$[117,135,0]$} & $\mathrm{e}$ & $\mathbb{Q}(\sqrt{57})$ \\
\hline$q_{10}$ & 10 & 1.35610 & 20 & {$[130,121,1]$} & $\mathrm{e}$ & $\mathbb{Q}(\sqrt{13})$ \\
\hline$r_{10}$ & 10 & 1.35994 & 8 & {$[131,120,1]$} & e & $\mathbb{Q}(\sqrt{13})$ \\
\hline$s_{10}$ & 10 & 1.36331 & 8 & {$[126,125,1]$} & $\mathrm{e}$ & $\operatorname{deg} 3$ \\
\hline$t_{10}$ & 10 & 1.36788 & 4 & {$[119,132,1]$} & $\mathrm{e}$ & $\operatorname{deg} 4$ \\
\hline$u_{10}$ & 10 & 1.37437 & 4 & {$[110,141,1]$} & $\mathrm{e}$ & $\mathbb{Q}(\sqrt{193})$ \\
\hline$v_{10}$ & 10 & 1.38201 & 16 & {$[102,149,1]$} & $\mathrm{e}$ & $\mathbb{Q}(\sqrt{73})$ \\
\hline$w_{10}$ & 10 & 1.38316 & 1440 & {$[90,162,0]$} & e $\left(A_{5}^{2}=\left(A_{5}^{3}\right)^{*}\right)$ & $\mathbb{Q}$ \\
\hline$x_{10}$ & 10 & 1.39022 & 96 & {$[114,136,2]$} & we & $\mathbb{Q}$ \\
\hline$y_{10}$ & 10 & 1.39466 & 16 & {$[98,152,2]$} & {$\left[\begin{array}{ll}t_{11} & \text { we }\end{array}\right]$} & \\
\hline
\end{tabular}


Table 3 (continued)

\begin{tabular}{|c|c|c|c|c|c|c|}
\hline class & $\mathbf{s}$ & $\gamma$ & g & Spect & Eut & Field \\
\hline$a_{9}$ & 9 & 1,21673 & 32 & {$[86,40,0]$} & e $\left(\mathrm{E}\left(b_{8}^{4}\right) \perp A_{1}\right)$ & $\mathbb{Q}$ \\
\hline$b_{9}$ & 9 & 1.21673 & 576 & {$[78,48,0]$} & $\left(A_{3} \perp A_{2}\right)$ & $\mathbb{Q}$ \\
\hline$c_{9}$ & 9 & 1.23499 & 32 & {$[81,45,0]$} & e $\left(\mathrm{E}\left(a_{8}^{4}\right) \perp A_{1}\right)$ & $\mathbb{Q}(\sqrt{3})$ \\
\hline$d_{9}$ & 9 & 1.25878 & 96 & {$[72,54,0]$} & e & $\mathbb{Q}$ \\
\hline$e_{9}$ & 9 & 1.25878 & 16 & {$[71,55,0]$} & e & $\mathbb{Q}$ \\
\hline$f_{9}$ & 9 & 1.25878 & 32 & {$[74,52,0]$} & se & $\mathbb{Q}$ \\
\hline$g_{9}$ & 9 & 1.25878 & 768 & {$[78,48,0]$} & we & $\mathbb{Q}$ \\
\hline$h_{9}$ & 9 & 1.27542 & 48 & {$[70,56,0]$} & e & $\mathbb{Q}$ \\
\hline$i_{9}$ & 9 & 1.28603 & 32 & {$[66,60,0]$} & $\mathrm{e}$ & $\mathbb{Q}(\sqrt{13})$ \\
\hline$j_{9}$ & 9 & 1.28603 & 8 & {$[65,61,0]$} & e & $\mathbb{Q}(\sqrt{13})$ \\
\hline$k_{9}$ & 9 & 1.28603 & 16 & {$[62,64,0]$} & $\mathrm{e}$ & $\mathbb{Q}(\sqrt{13})$ \\
\hline$l_{9}$ & 9 & 1.28878 & 64 & {$[62,64,0]$} & {$\left[\begin{array}{ll}f_{10} & \text { we }\end{array}\right]$} & \\
\hline$m_{9}$ & 9 & 1.30136 & 8 & {$[60,66,0]$} & e & $\mathbb{Q}$ \\
\hline$n_{9}$ & 9 & 1.30734 & 8 & {$[57,69,0]$} & e & $\operatorname{deg} 4$ \\
\hline$o_{9}$ & 9 & 1.32108 & 24 & {$[51,75,0]$} & e & $\mathbb{Q}(\sqrt{6})$ \\
\hline$p_{9}$ & 9 & 1.33790 & 144 & {$[45,81,0]$} & $\mathrm{e}$ & $\mathbb{Q}$ \\
\hline$q_{9}$ & 9 & 1.34540 & 96 & {$[65,60,1]$} & $\mathrm{e}$ & $\mathbb{Q}$ \\
\hline$r_{9}$ & 9 & 1.34978 & 8 & {$[59,66,1]$} & $\mathrm{e}$ & $\mathbb{Q}(\sqrt{73})$ \\
\hline$s_{9}$ & 9 & 1.35401 & 64 & {$[57,68,1]$} & $\mathrm{e}$ & $\mathbb{Q}$ \\
\hline$t_{9}$ & 9 & 1.35492 & 24 & {$[57,68,1]$} & $\mathrm{e}$ & $\mathbb{Q}(\sqrt{41})$ \\
\hline$u_{9}$ & 9 & 1.35815 & 8 & {$[53,72,1]$} & $\mathrm{e}$ & $\operatorname{deg} 3$ \\
\hline$v_{9}$ & 9 & 1.36650 & 8 & {$[45,80,1]$} & $\mathrm{e}$ & $\operatorname{deg} 5$ \\
\hline$w_{9}$ & 9 & 1.39022 & 96 & {$[53,72,1]$} & {$\left[\begin{array}{ll}x_{10} & \text { we }\end{array}\right]$} & \\
\hline$a_{8}$ & 8 & 1.14870 & 384 & {$[40,16,0]$} & $\left(A_{3} \perp A_{1}^{2}\right)$ & $\mathbb{Q}$ \\
\hline$b_{8}$ & 8 & 1.18840 & 192 & {$[32,24,0]$} & e $\left(\mathrm{E}\left(a_{5}^{3}\right) \perp A_{2}\right)$ & $\mathbb{Q}$ \\
\hline$c_{8}$ & 8 & 1.18840 & 32 & {$[35,21,0]$} & e $\left(\mathrm{E}\left(c_{7}^{4}\right) \perp A_{1}\right)$ & $\mathbb{Q}$ \\
\hline$d_{8}$ & 8 & 1.18840 & 192 & {$[36,20,0]$} & se $\left(\mathrm{E}\left(b_{7}^{4}\right) \perp A_{1}\right)$ & $\mathbb{Q}$ \\
\hline$e_{8}$ & 8 & 1.21412 & 32 & {$[32,24,0]$} & e $\left(\mathrm{E}\left(a_{7}^{4}\right) \perp A_{1}\right)$ & $\mathbb{Q}(\sqrt{13})$ \\
\hline$f_{8}$ & 8 & 1.24573 & 96 & {$[28,28,0]$} & $\mathrm{e}$ & $\mathbb{Q}$ \\
\hline$g_{8}$ & 8 & 1.24573 & 32 & {$[26,30,0]$} & $\mathrm{e}$ & $\mathbb{Q}$ \\
\hline$h_{8}$ & 8 & 1.24573 & 24 & {$[27,29,0]$} & $\mathrm{e}$ & $\mathbb{Q}$ \\
\hline$i_{8}$ & 8 & 1.25878 & 32 & {$[24,32,0]$} & {$\left[\begin{array}{ll}f_{9} & \mathrm{se}\end{array}\right]$} & \\
\hline$j_{8}$ & 8 & 1.25878 & 768 & {$[24,32,0]$} & {$\left[\begin{array}{ll}g_{9} & \text { we }\end{array}\right]$} & \\
\hline$k_{8}$ & 8 & 1.26867 & 48 & {$[24,32,0]$} & $\mathrm{e}$ & $\mathbb{Q}(\sqrt{33})$ \\
\hline$l_{8}$ & 8 & 1.28160 & 16 & {$[21,35,0]$} & $\mathrm{e}$ & $\mathbb{Q}(\sqrt{7})$ \\
\hline$m_{8}$ & 8 & 1.28428 & 64 & {$[20,36,0]$} & $\mathrm{e}$ & $\mathbb{Q}(\sqrt{5})$ \\
\hline$n_{8}$ & 8 & 1.34027 & 24 & {$[23,32,1]$} & e & $\mathbb{Q}(\sqrt{241})$ \\
\hline$O_{8}$ & 8 & 1.34492 & 16 & {$[20,35,1]$} & $\mathrm{e}$ & $\mathbb{Q}(\sqrt{3})$ \\
\hline$p_{8}$ & 8 & 1.35434 & 24 & {$[15,40,1]$} & $\mathrm{e}$ & $\operatorname{deg} 3$ \\
\hline
\end{tabular}


Table 3 (continued)

\begin{tabular}{|r|c|c|r|r|rr|r|}
\hline class & s & $\gamma$ & \multicolumn{1}{|c|}{ g } & Spect & \multicolumn{1}{c|}{ Eut } & \multicolumn{1}{c|}{ Field } \\
\hline$a_{7}$ & 7 & 1.12195 & 128 & {$[13,8,0]$} & $\mathrm{e}\left(\mathrm{E}\left(a_{5}^{3}\right) \perp A_{1}^{2}\right)$ & $\mathbb{Q}$ \\
$b_{7}$ & 7 & 1.12195 & 576 & {$[12,9,0]$} & $\mathrm{e}$ & $\left(A_{2}^{2} \perp A_{1}\right)$ & $\mathbb{Q}$ \\
$c_{7}$ & 7 & 1.17607 & 576 & {$[9,12,0]$} & $\mathrm{e}\left(A_{2} \perp A_{3} *\right)$ & $\mathbb{Q}$ \\
$d_{7}$ & 7 & 1.17607 & 48 & {$[10,11,0]$} & $\mathrm{e}\left(\mathrm{E}\left(b_{6}^{4}\right) \perp A_{1}\right)$ & $\mathbb{Q}$ \\
$e_{7}$ & 7 & 1.18840 & 192 & {$[9,12,0]$} & - & {$\left[d_{8} \mathrm{se}\right]$} & $\mathbb{Q}$ \\
$f_{7}$ & 7 & 1.22251 & 96 & {$[7,14,0]$} & $\mathrm{e}$ & & $\mathbb{Q}$ \\
$g_{7}$ & 7 & 1.23281 & 144 & {$[6,15,0]$} & $\mathrm{e}$ & & $\mathbb{Q}(\sqrt{89})$ \\
$h_{7}$ & 7 & 1.24501 & 96 & {$[5,16,0]$} & $\mathrm{e}$ & & $\mathbb{Q}$ \\
$i_{7}$ & 7 & 1.33211 & 96 & {$[6,14,1]$} & $\mathrm{e}$ & & $\mathbb{Q}(\sqrt{409})$ \\
$j_{7}$ & 7 & 1.33746 & 48 & {$[4,16,1]$} & $\mathrm{e}$ & \\
\hline$a_{6}$ & 6 & 1.05922 & 576 & {$[3,3,0]$} & $\mathrm{e}$ & $\left(A_{2} \perp A_{1}^{3}\right)$ & $\mathbb{Q}$ \\
$b_{6}$ & 6 & 1.11032 & 384 & {$[2,4,0]$} & $\mathrm{e}$ & $\left(A_{3}^{*} \perp A_{1}^{2}\right)$ & $\mathbb{Q}$ \\
$c_{6}$ & 6 & 1.15415 & 480 & {$[1,5,0]$} & $\mathrm{e}$ & $\left(A_{4}^{*} \perp A_{1}\right)$ & $\mathbb{Q}$ \\
$d_{6}$ & 6 & 1.19247 & 1440 & {$[0,6,0]$} & $\mathrm{e}$ & $\left(A_{5}^{*}\right)$ & $\mathbb{Q}$ \\
$e_{6}$ & 6 & 1.32691 & 240 & {$[0,5,1]$} & $\mathrm{e}$ & & $\mathbb{Q}$ \\
\hline$a_{5}$ & 5 & 1.00000 & 3840 & {$[0,1,0]$} & $\mathrm{e}$ & $\left(A_{1}^{5}\right)$ & $\mathbb{Q}$ \\
$b_{5}$ & 5 & 1.31950 & 3840 & {$[0,0,1]$} & $\mathrm{e}$ & $\left(D_{5}^{*}\right)$ & $\mathbb{Q}$ \\
\hline
\end{tabular}


TABLE 4. This table gives the upper entries $\alpha_{i j}$ of the rational weakly-eutactic matrices in the corresponding class.

\begin{tabular}{|c|c|c|c|c|c|c|c|c|c|c|c|c|c|c|c|}
\hline ass & $\alpha_{11}$ & $\alpha_{12}$ & $\alpha_{13}$ & $\alpha_{14}$ & $\alpha_{15}$ & $\alpha_{22}$ & $\alpha_{23}$ & $\alpha_{24}$ & $\alpha_{25}$ & $\alpha_{33}$ & $\alpha_{34}$ & $\alpha_{35}$ & $\alpha_{44}$ & $\alpha_{45}$ & $\alpha_{55}$ \\
\hline$A_{5}$ & 2 & 1 & 1 & 1 & 1 & 2 & 1 & 1 & 1 & 2 & 1 & 1 & 2 & 1 & 2 \\
\hline$A_{5}^{3}$ & 4 & 1 & 2 & 2 & 2 & 4 & 2 & 2 & 2 & 4 & 1 & 1 & 4 & 1 & 4 \\
\hline$D_{5}$ & 2 & 0 & 1 & 1 & 1 & 2 & 1 & 1 & 1 & 2 & 1 & 1 & 2 & 1 & 2 \\
\hline$a_{14}$ & 8 & 3 & 4 & 4 & 4 & 8 & 4 & 4 & 4 & 8 & 4 & 4 & 8 & 4 & 8 \\
\hline$b_{14}$ & 4 & 1 & 2 & 2 & 2 & 4 & 2 & 2 & 2 & 4 & 2 & 2 & 4 & 1 & 4 \\
\hline$d_{14}$ & 4 & 0 & 1 & 2 & 2 & 4 & 2 & 2 & 2 & 4 & 2 & 2 & 4 & 2 & 4 \\
\hline$a_{13}$ & 6 & 3 & 3 & 3 & 3 & 6 & 3 & 3 & 4 & 6 & 3 & 4 & 6 & 4 & 8 \\
\hline$c_{13}$ & 4 & 0 & 2 & 1 & 1 & 4 & 2 & 2 & 2 & 4 & 1 & 2 & 4 & 2 & 4 \\
\hline$f_{13}$ & 16 & 4 & 9 & 8 & 8 & 16 & 9 & 8 & 8 & 18 & 9 & 9 & 16 & 4 & 16 \\
\hline$g_{13}$ & 6 & 0 & 3 & 4 & 2 & 6 & 3 & 3 & 3 & 6 & 3 & 3 & 8 & 4 & 6 \\
\hline$i_{13}$ & 6 & 0 & 3 & 3 & 2 & 6 & 3 & 3 & 4 & 6 & 3 & 4 & 6 & 4 & 8 \\
\hline$a_{12}$ & 4 & 2 & 2 & 2 & 2 & 4 & 2 & 2 & 2 & 4 & 2 & 3 & 4 & 3 & 6 \\
\hline$b_{12}$ & 8 & 4 & 4 & 4 & 4 & 6 & 3 & 3 & 4 & 6 & 3 & 4 & 6 & 4 & 8 \\
\hline$c_{12}$ & 18 & 9 & 9 & 12 & 10 & 18 & 9 & 12 & 13 & 18 & 12 & 13 & 24 & 16 & 26 \\
\hline$e_{12}$ & 4 & 2 & 2 & 2 & 3 & 6 & 3 & 3 & 4 & 4 & 2 & 3 & 4 & 2 & 6 \\
\hline$f_{12}$ & 10 & 5 & 5 & 4 & 6 & 10 & 4 & 5 & 6 & 10 & 5 & 6 & 10 & 6 & 12 \\
\hline$k_{12}$ & 8 & 1 & 4 & 4 & 3 & 8 & 4 & 3 & 4 & 8 & 4 & 4 & 8 & 1 & 8 \\
\hline$l_{12}$ & 4 & 0 & 2 & 2 & 2 & 4 & 2 & 2 & 2 & 4 & 2 & 3 & 4 & 3 & 6 \\
\hline$p_{12}$ & 10 & -5 & -5 & 4 & 2 & 10 & 4 & -5 & 2 & 10 & 1 & -4 & 10 & -4 & 10 \\
\hline$a_{11}$ & 2 & 1 & 1 & 1 & 0 & 2 & 1 & 1 & 0 & 2 & 1 & 0 & 2 & 0 & 2 \\
\hline$b_{11}$ & 2 & 1 & -1 & 1 & 0 & 2 & -1 & 1 & 0 & 2 & 0 & 0 & 2 & 0 & 2 \\
\hline$c_{11}$ & 12 & 6 & 6 & 8 & 7 & 12 & 6 & 8 & 7 & 12 & 8 & 10 & 16 & 12 & 20 \\
\hline$d_{11}$ & 4 & 2 & 2 & 2 & 2 & 4 & 2 & 3 & 3 & 4 & 3 & 3 & 6 & 4 & 6 \\
\hline$e_{11}$ & 12 & 6 & 6 & 8 & 6 & 12 & 6 & 8 & 9 & 12 & 8 & 9 & 16 & 8 & 18 \\
\hline$f_{11}$ & 8 & 5 & 4 & 6 & 6 & 14 & 7 & 9 & 9 & 8 & 6 & 6 & 12 & 6 & 12 \\
\hline$p_{11}$ & 100 & 25 & 50 & 60 & 51 & 100 & 50 & 60 & 66 & 100 & 60 & 66 & 120 & 36 & 132 \\
\hline$r_{11}$ & 10 & 2 & 5 & 4 & 6 & 10 & 4 & 5 & 6 & 10 & 5 & 6 & 10 & 3 & 12 \\
\hline$v_{11}$ & 8 & -4 & -3 & 3 & 2 & 8 & 3 & -3 & 2 & 8 & 2 & -3 & 8 & -3 & 8 \\
\hline$a_{10}$ & 6 & 3 & 3 & -2 & 0 & 6 & 3 & 1 & 0 & 6 & 1 & 0 & 6 & 0 & 6 \\
\hline$b_{10}$ & 4 & 0 & 0 & 0 & 0 & 4 & 2 & -1 & 2 & 4 & 1 & 1 & 4 & 1 & 4 \\
\hline$c_{10}$ & 4 & 2 & 3 & 3 & 3 & 4 & 3 & 3 & 3 & 6 & 4 & 5 & 6 & 5 & 8 \\
\hline$d_{10}$ & 8 & 6 & 4 & 6 & 5 & 12 & 6 & 8 & 7 & 8 & 6 & 7 & 12 & 9 & 14 \\
\hline$e_{10}$ & 8 & 4 & 4 & 4 & 4 & 8 & 4 & 6 & 5 & 8 & 6 & 7 & 12 & 9 & 14 \\
\hline$f_{10}$ & 4 & 2 & 2 & 2 & 2 & 4 & 2 & 3 & 3 & 4 & 3 & 3 & 6 & 3 & 6 \\
\hline$o_{10}$ & 12 & 3 & 6 & 4 & 7 & 12 & 6 & 6 & 9 & 12 & 3 & 9 & 12 & 2 & 18 \\
\hline$w_{10}$ & 3 & 1 & 1 & -1 & -1 & 3 & -1 & 1 & -1 & 3 & -1 & 1 & 3 & 1 & 3 \\
\hline$x_{10}$ & 12 & 1 & 6 & 5 & 5 & 12 & 6 & 5 & 5 & 12 & 6 & 6 & 12 & 1 & 12 \\
\hline$a_{9}$ & 4 & 2 & 2 & 0 & 0 & 4 & 2 & -1 & 0 & 4 & 1 & 0 & 4 & 0 & 4 \\
\hline$b_{9}$ & 2 & 1 & 1 & 0 & 0 & 2 & 1 & 0 & 0 & 2 & 0 & 0 & 2 & 1 & 2 \\
\hline$d_{9}$ & 14 & 7 & 5 & 9 & 7 & 8 & 4 & 6 & 5 & 8 & 6 & 7 & 12 & 9 & 14 \\
\hline$e_{9}$ & 24 & 12 & 12 & 18 & 15 & 16 & 8 & 10 & 9 & 16 & 14 & 15 & 28 & 21 & 30 \\
\hline$f_{9}$ & 12 & 9 & 6 & 6 & 6 & 14 & 7 & 5 & 6 & 8 & 4 & 6 & 8 & 6 & 12 \\
\hline$g_{9}$ & 6 & 3 & 3 & 3 & 3 & 4 & 2 & 3 & 3 & 4 & 3 & 3 & 6 & 3 & 6 \\
\hline$h_{9}$ & 6 & 3 & 3 & 3 & 3 & 6 & 3 & 5 & 4 & 6 & 4 & 5 & 10 & 7 & 10 \\
\hline$m_{9}$ & 36 & 18 & 12 & 24 & 18 & 36 & 18 & 24 & 21 & 36 & 20 & 28 & 48 & 34 & 56 \\
\hline$p_{9}$ & 6 & 3 & 3 & 3 & 3 & 5 & 2 & 2 & 3 & 5 & 2 & 3 & 5 & 3 & 6 \\
\hline$q_{9}$ & 6 & 0 & 2 & 2 & 3 & 6 & 3 & 3 & 3 & 6 & 3 & 4 & 6 & 1 & 8 \\
\hline$s_{9}$ & 8 & 2 & 5 & 5 & 5 & 8 & 5 & 5 & 5 & 10 & 6 & 7 & 10 & 3 & 12 \\
\hline
\end{tabular}


Table 4 (continued)

$\begin{array}{cccccccccccccccc}\text { class } & \alpha_{11} & \alpha_{12} & \alpha_{13} & \alpha_{14} & \alpha_{15} & \alpha_{22} & \alpha_{23} & \alpha_{24} & \alpha_{25} & \alpha_{33} & \alpha_{34} & \alpha_{35} & \alpha_{44} & \alpha_{45} & \alpha_{55} \\ a_{8} & 2 & 1 & 1 & 0 & 0 & 2 & 1 & 0 & 0 & 2 & 0 & 0 & 2 & 0 & 2 \\ b_{8} & 4 & 2 & 0 & 0 & 0 & 4 & 0 & 0 & 0 & 4 & 2 & -1 & 4 & 1 & 4 \\ c_{8} & 8 & 4 & -2 & 1 & 0 & 8 & 2 & -1 & 0 & 8 & 2 & 0 & 8 & 0 & 8 \\ d_{8} & 4 & 2 & -1 & -1 & 0 & 4 & 1 & 1 & 0 & 4 & 1 & 0 & 4 & 0 & 4 \\ f_{8} & 18 & 9 & 9 & 9 & 9 & 12 & 6 & 10 & 8 & 12 & 8 & 10 & 20 & 14 & 20 \\ g_{8} & 12 & 6 & 8 & 10 & 9 & 12 & 10 & 8 & 9 & 20 & 14 & 17 & 20 & 17 & 26 \\ h_{8} & 12 & 6 & 9 & 7 & 8 & 12 & 9 & 11 & 10 & 18 & 12 & 15 & 22 & 17 & 24 \\ a_{7} & 4 & 2 & -1 & 0 & 0 & 4 & 1 & 0 & 0 & 4 & 0 & 0 & 4 & 0 & 4 \\ b_{7} & 2 & 1 & 0 & 0 & 0 & 2 & 0 & 0 & 0 & 2 & 1 & 0 & 2 & 0 & 2 \\ c_{7} & 6 & 3 & 0 & 0 & 0 & 6 & 0 & 0 & 0 & 6 & 2 & -2 & 6 & 2 & 6 \\ d_{7} & 6 & 3 & -1 & 1 & 0 & 6 & 1 & -1 & 0 & 6 & 2 & 0 & 6 & 0 & 6 \\ f_{7} & 8 & 4 & 7 & 5 & 6 & 8 & 5 & 7 & 6 & 14 & 8 & 11 & 14 & 11 & 16 \\ g_{7} & 9 & 3 & 6 & 4 & 5 & 9 & 6 & 8 & 7 & 12 & 8 & 10 & 16 & 12 & 17 \\ i_{7} & 24 & 0 & 9 & 11 & 11 & 24 & 12 & 12 & 12 & 24 & 15 & 15 & 30 & 4 & 30 \\ a_{6} & 2 & 1 & 0 & 0 & 0 & 2 & 0 & 0 & 0 & 2 & 0 & 0 & 2 & 0 & 2 \\ b_{6} & 3 & 1 & -1 & 0 & 0 & 3 & 1 & 0 & 0 & 3 & 0 & 0 & 3 & 0 & 3 \\ c_{6} & 4 & 1 & -1 & 1 & 0 & 4 & 1 & -1 & 0 & 4 & 1 & 0 & 4 & 0 & 4 \\ d_{6} & 5 & -1 & -1 & -1 & -1 & 5 & -1 & -1 & -1 & 5 & -1 & -1 & 5 & -1 & 5 \\ e_{6} & 20 & 1 & 9 & 10 & 10 & 20 & 12 & 11 & 11 & 24 & 15 & 15 & 26 & 5 & 26 \\ a_{5} & 5 & 3 & -2 & -2 & -2 & 5 & -2 & -2 & -2 & 4 & 0 & 0 & 4 & 0 & 4 \\ b_{5} & 1 & 0 & 0 & 0 & 0 & 1 & 0 & 0 & 0 & 1 & 0 & 0 & 1 & 0 & 1\end{array}$


Acknowledgments. I gratefully acknowledge the helpful discussions that I have had with Anne-Marie Bergé and Christophe Bavard. I also wish to thank Jacques Martinet for his encouragement and Pierrette Cassou-Noguès for her help in Maple handling, and the other authors of the PARI system. I am grateful to the referee for suggesting improvements and for correcting errors I made.

\section{REFERENCES}

[Ash1] A.Ash, On eutactic forms, Can. J. Math. 29 (1977), 1040-1054. MR 58:10762

[Ash2] A.Ash, On the existence of eutactic forms, Bull. London Math. Soc. 12 (1980), 192-196. MR 83a:10034

[Ba] J.-L. Baril, Autour de l'algorithme de Voronoi: constructions de réseaux euclidiens, Thèse Bordeaux (1996).

[Bv] C. Bavard, Une formule d'Euler pour les classes minimales de réseaux, preprint.

[BM] A.-M. Bergé, J. Martinet, Sur la classification des réseaux eutactiques, J. London Math. Soc., 53 ((1996)), 417-432. MR 97i:11076

[CS] J.H. Conway, N.J.A. Sloane, Sphere Packings, Lattices and Groups, Springer-Verlag, Grundlehren n²290, Heidelberg, 1988. MR 89a:11067

[CS1] J.H. Conway, N.J.A. Sloane, Low-dimensional lattices. III. Perfect forms, Proc. Royal Soc. London A 418 (1988), 43-80. MR 90a:11073

[KZ3] A. Korkine, G. Zolotareff, Sur les formes quadratiques positives, Math. Ann. 11 (1877), 242-292.

[Mar] J. Martinet, Les réseaux parfaits des espaces euclidiens, Masson, Paris, 1996. MR 98a:11086

[PS] W. Plesken, B. Souvignier, Computing isometries of lattices, J. Symb. Comp. 24 (1997) 327-354. MR 98i:11047

[Sto] W.I. Štogrin, Quasi densest lattice packing of spheres., Dokl-Akad-Nauk-SSSR 218 (1974), $62-65$.

[Vor] G. Voronoï, Nouvelles applications des paramètres continus à la théorie des formes quadratiques : 1 Sur quelques propriétés des formes quadratiques positives parfaites, J. Reine Angew. Math 133 (1908), 97-178.

[W] G.L. Watson, On the minimum points of a positive quadratic form, Mathematica 18 (1971), 60-70. MR 44:6612

A2X, Mathématiques, Université Bordeaux I, 351, Cours de la Libération, 33405 Talence cedex, France

E-mail address: christian.batut@math.u-bordeaux.fr 